\title{
Mutations in the bacterial cell division protein FtsZ highlight the role of GTP binding and longitudinal subunit interactions in assembly and function
}

\author{
Heidi A. Arjes ${ }^{1,2}$, Bradley Lai ${ }^{1}$, Ezinwanne Emelue ${ }^{1}$, Adriana Steinbach ${ }^{1}$ and Petra Anne Levin ${ }^{1 *}$
}

\begin{abstract}
Background: Assembly of the tubulin-like GTPase, FtsZ, at the future division site initiates the process of bacterial cytokinesis. The FtsZ ring serves as a platform for assembly of the division machinery and constricts at the leading edge of the invaginating septum during cytokinesis. In vitro, FtsZ assembles in a GTP-dependent manner, forming straight filaments that curve upon GTP hydrolysis. FtsZ binds but cannot hydrolyze GTP as a monomer. Instead, the active site for GTP hydrolysis is formed at the monomer-monomer interface upon dimerization. While the dynamics of GTP hydrolysis and assembly have been extensively studied in vitro, significantly less is known about the role of GTP binding and hydrolysis in vivo. ftsZ84, a GTPase defective allele of Escherichia coli ftsZ, provides a striking example of the disconnect between in vivo and in vitro FtsZ assembly.
\end{abstract}

Results: Although ftsZ84 mutants are defective for FtsZ ring formation and division under nonpermissive conditions, they are near wild type for ring formation and division under permissive conditions. In vitro, however, purified FtsZ84 is defective in GTP binding, hydrolysis and assembly under standard reaction conditions. To clarify the nature of the FtsZ84 assembly defect, we isolated and characterized three intragenic suppressors of ftsZ84. All three suppressor mutations increased the apparent affinity of FtsZ84 for GTP, consistent with improved subunit-subunit interactions along the longitudinal interface. Although kinetic analysis indicates that the suppressor mutations increase the affinity of FtsZ84 for GTP, all three exhibit reduced rates of GTP hydrolysis and fail to support assembly in vitro.

Conclusion: Together, our data suggest that FtsZ, and potentially other enzymes whose assembly is similarly regulated, can compensate for defects in catalysis through increases in substrate binding and subunit-subunit interactions. In addition, these results highlight the dichotomy between commonly used in vitro assembly conditions and FtsZ ring formation in the complex intracellular milieu.

Keywords: FtsZ, Cell division, Cytoskeleton, Protein assembly, GTPase activity, FtsZ assembly

\section{Background}

Assembly of the tubulin-like GTPase FtsZ at the future division site is a fundamental step in bacterial cytokinesis $[1,2]$. In response to an unidentified cell cycle signal, FtsZ transitions from primarily cytoplasmic monomers and/or short polymers into the longer polymers that constitute the FtsZ ring and serve as the foundation for assembly of

\footnotetext{
*Correspondence: plevin@wustl.edu

'Department of Biology, Washington University in St. Louis, St. Louis, MO 63130, USA

Full list of author information is available at the end of the article
}

the division machinery [2]. The FtsZ ring is highly dynamic, with subunit turnover rates on the order of seconds [2]. Although subunit turnover is high, the ring itself is present for $\sim 80 \%$ of the division cycle under nutrient rich conditions $[3,4]$. Why the ring remains in place so long is unclear, although studies of cells defective in assembly of downstream components of the division machinery suggest a maturation process may be at work $[5,6]$. At the end of the division cycle, in response to another unidentified signal, the FtsZ ring constricts at the leading edge of the invaginating septum. Work 
from our laboratory and others indicates that FtsZ concentration is constant throughout the cell cycle and that the precise spatial and temporal regulation of bacterial cell division is governed by tightly orchestrated changes in FtsZ assembly dynamics $[3,4,7]$.

Like tubulin, multimerization is a prerequisite for FtsZ-mediated GTP hydrolysis. FtsZ binds to GTP as a monomer but cannot hydrolyze it as such. The active site for GTP hydrolysis is formed at the longitudinal interface between two FtsZ subunits where the GTPbinding pocket of one monomer contacts the T7 synergy loop of the adjacent monomer (Fig. 1a) [2, 8]. In vitro, GTP binding stimulates FtsZ assembly into single stranded polymers (also known as protofilaments). Higher order assembly of FtsZ protofilaments into polymer bundles is also possible in vitro, depending on buffer conditions, and, in some cases, the bacterial species from whence the FtsZ was derived $[9,10]$. GTP-bound FtsZ protofilaments are initially straight, curving only upon nucleotide hydrolysis [2]. Once GTP has been hydrolyzed to GDP, polymers can disassemble and exchange GDP for GTP and assemble once again [2].

Although GTP binding and hydrolysis have a strong impact on FtsZ assembly in vitro, their role in vivo is less clear. The disconnect between in vitro and in vivo data is exemplified by ftsZ84, a heat-sensitive allele of fts $Z$ encoding a point mutation in the GTP binding pocket (G105S) [11]. While ftsZ84 cells exhibit predominantly normal FtsZ ring formation and division under permissive conditions $\left(30^{\circ} \mathrm{C}\right.$ in $\mathrm{LB}$ with $\left.1 \% \mathrm{NaCl}\right)$, under nonpermissive conditions $\left(42{ }^{\circ} \mathrm{C}\right.$ in $\mathrm{LB}$ with no salt), ftsZ84 mutants are defective for FtsZ ring formation and division [12,13]. Surprisingly, although functional in vivo under permissive conditions, FtsZ84 protein is unable to assemble into protofilaments in vitro, regardless of temperature, and is severely defective in GTP binding and hydrolysis [14-16]. The inability of FtsZ84 to assemble in vitro when it can support division in vivo under permissive conditions raises questions about the relationship between in vitro FtsZ assembly and in vivo function. Differences between in vitro and in vivo assembly are likely due in part to limitations in our ability replicate the in vivo environment in vitro; not only in regard to buffer conditions, but also in regard to the host of modulatory proteins that are normally found in the cellular milieu. With regard to the latter, ZapA in particular has been found to promote lateral interactions and assembly of FtsZ in vitro [17].

To clarify the nature of the ftsZ84 defect at the functional level, we identified three intragenic suppressors that restored FtsZ ring formation and division to ftsZ84 mutants under nonpermissive conditions and GTP binding, but not polymerization, in vitro. Together our findings highlight a fundamental role for robust longitudinal

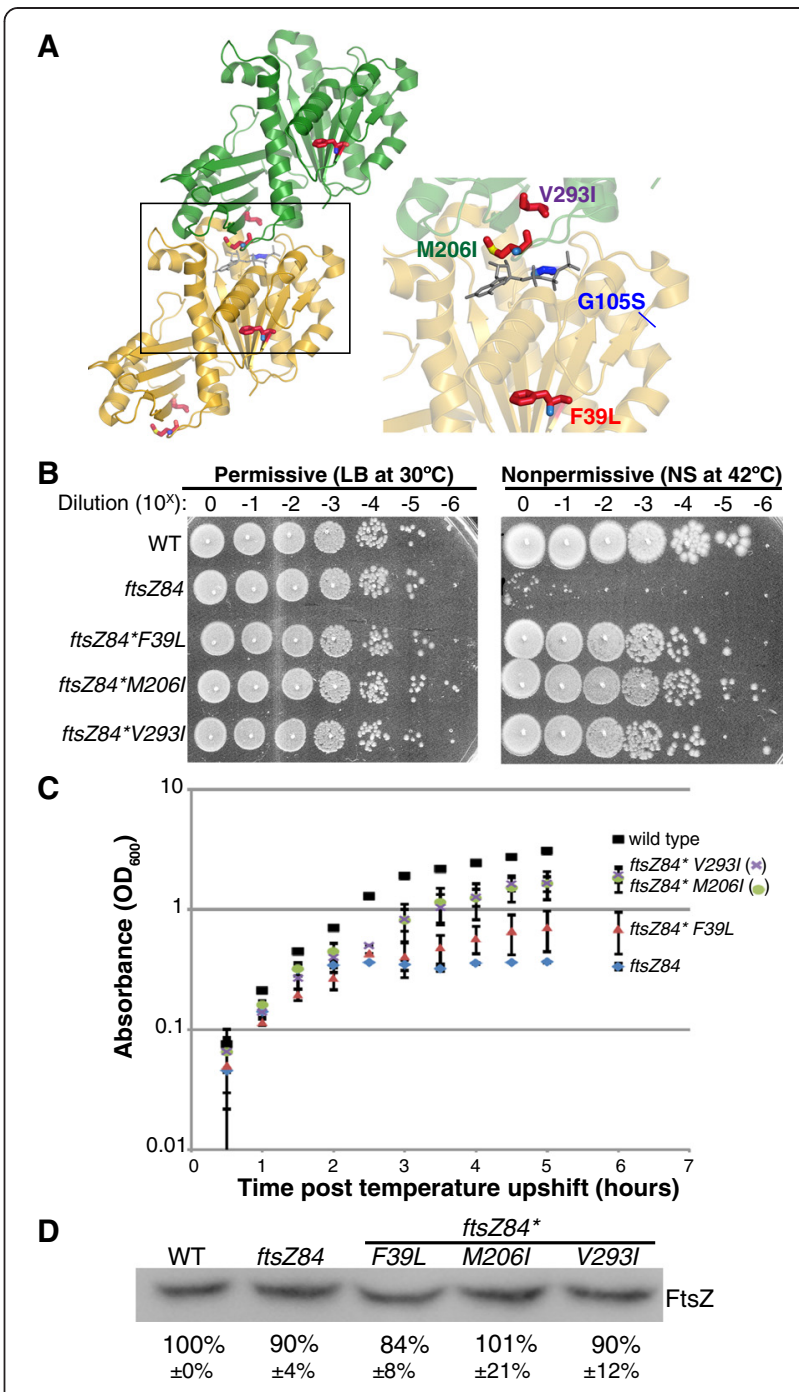

Fig. 1 Secondary mutations restore temperature resistance to ftsZ84 cells without increasing FtsZ concentration. a Location of the FtsZ84 (G105S) point mutation and the three intragenic suppressors of that mutation mapped onto an FtsZ dimer from Staphylococcus aureus. G10S5S (blue) is the original ftsZ84 point mutation. GDP is shown in gray. The three intragenic suppressors F39L, M206I and V293I are highlighted in the inset. The crystal structure is modified from the $S$. aureus FtsZ dimer structure (PDB ID: 3WGN) [52]. b Plating efficiency assays of wild-type, ftsZ84, and the three intragenic ftsZ84 suppressor strains. Tenfold dilutions of cells cultured in permissive conditions were plated under permissive and nonpermissive conditions. This experiment was repeated 3 times with identical results. One representative experiment is shown. c Growth as measured by absorbance $\left(\mathrm{OD}_{600}\right)$ of wild-type, $\mathrm{fts} Z 84$, and the three intragenic ftsZ84 suppressor strains under nonpermissive conditions. Error bars represent standard deviation of 3 independent experiments. d Quantitative immunoblot indicates that intracellular FtsZ concentrations are wild type in fts $Z 84$ and fts $Z 84^{*}$ mutants under nonpermissive conditions. After $\sim 3$ mass doubling periods in LB no salt, cells were sampled at equivalent OD's to ensure the same amount of protein was loaded per lane and protein bands were normalized to total protein (Ponceau staining) as a loading and transfer control. ImageJ software was used to quantify band intensity. The average and standard deviation of 3 independent experiments are shown below the blot 
subunit interactions in assembly of FtsZ protofilaments and suggest these interactions are reinforced in vivo through the actions of multiple FtsZ modulatory proteins.

\section{Results}

Identification of intragenic suppressors of ftsZ84

To identify spontaneous suppressor mutations that restore the ability of ftsZ84 mutants to grow under nonpermissive conditions, we cultured ftsZ84 cells to mid-exponential phase $\left(\mathrm{OD}_{600}=0.2-0.5\right)$ under permissive conditions $\left(30{ }^{\circ} \mathrm{C}, \mathrm{LB} 1 \% \mathrm{NaCl}\right)$, then washed and plated cells on no salt medium at $42{ }^{\circ} \mathrm{C}$ overnight. Under these restrictive conditions, ftsZ84 mutants were reduced more than $10^{5}$-fold for colony formation (Fig. 1b). Any colonies that arose after overnight incubation were the result of spontaneous suppressor mutations. Suppressors were isolated at a frequency of $1.5^{*} 10^{-5}$ consistent with the majority representing loss-of-function mutations in average size $(\sim 1 \mathrm{~kb})$ genes. We also employed ethyl methanesulfonate (EMS) mutagenesis in a second screen in an attempt to identify additional intragenic suppressor mutations. EMS alkylates guanine and commonly causes thymine to pair with the alkylated guanine residues causing $\mathrm{G}: \mathrm{C}$ to $\mathrm{A}: \mathrm{T}$ substitutions.

To specifically identify those suppressor mutations that were intragenic, we employed $\mathrm{P} 1$ phage transduction to determine the linkage between individual mutations and the $f t s Z 84$ allele $[8,9,15,18]$. Briefly, we transduced genomic DNA from the suppressors into wild-type $E$. coli and selected for tetracycline resistance (the ftsZ84 mutation is $55 \%$ linked to a tetracycline resistant (TetR) Tn10 insertion in the leuD gene) [18]. The resulting colonies were analyzed for temperature sensitivity (denoting the suppressor mutation was not linked to ftsZ84) or temperature resistance (denoting the suppressor mutation was linked to ftsZ84).

7 of 96 spontaneous suppressor mutations and 10 of 14 EMS generated suppressor mutations were linked to fts $Z$ by P1 transduction. Sequencing revealed that all linked mutations were secondary mutations within the ftsZ84 coding sequence. The remaining 93 suppressor mutations did not co-transduce with ftsZ84 suggesting they are located in other chromosomal regions distal to fts $Z$ and the Tn10::tetR marker.

We isolated three different intragenic suppressor mutations within the fts $Z$ open reading frame: a $\mathrm{T}$ to $\mathrm{C}$ transition at base pair 115 that changes a phenylalanine to a leucine (F39L), a G to A transition at base pair 618 that changes a methionine to an isoleucine (M206I) and a $\mathrm{G}$ to $\mathrm{A}$ transition at base pair 877 that changes a valine to an isoleucine (V293I) (Fig. 1a). For simplicity, all intragenic suppressors are designated ftsZ84* to indicate the presence of the original G105S mutation and a second intragenic suppressor mutation. The F39L mutation is located in the core of FtsZ and presumably impacts the structure of the active site (Fig. 1a). ftsZ84*F39L is phenotypically the weakest ftsZ84 suppressor with regard to growth rate and cell size under nonpermissive conditions (Fig. 1c, 2). By contrast, M206I and V293I are nearer to wild type with regard to both growth and cell size under the same conditions. M206I is located in the T7synergy loop, a region that contacts the GTP binding site in adjacent monomers and is essential for GTP hydrolysis (Fig. 1). ftsZ84*M206I cells restore length approximately twice as well as F39L at nonpermissive conditions (Fig. 2). V293I is located on the longitudinal subunit-subunit interface, and like M206I, presumably stabilizes interactions between monomers in FtsZ protofilaments (Fig. 1). Like ftsZ84*M206I mutants, ftsZ84*V293I cells restore cell length under nonpermissive conditions (Fig. 2).

Although we screened $\sim 10^{8}$ cells in 5 independent screens (4 screens for spontaneous suppressors and 1 EMS screen), we repeatedly isolated the same three intragenic suppressors of $f t s Z 84$. In screens for spontaneous suppressor mutations we isolated the F39L mutation twice, the M206I mutation 3 times and the V293I mutation twice. Using EMS, we isolated M206I once and identified 9 independent V293I mutations. (The nature of EMS mutagenesis precluded the identification of F39L, which requires a $\mathrm{T}$ to $\mathrm{C}$ transition.) Identification of such a limited number of suppressors is consistent with a strong relationship between primary sequence and FtsZ function in vivo.

Notably, transduction of the three fts $Z 84 *$ alleles ftsZ84*F39L, ftsZ84*M206I, and ftsZ84*V293I - into a fresh MG1655 wild-type background indicated that all three intragenic suppressor mutations are sufficient to fully restore colony forming ability under nonpermissive conditions. All the intragenic suppressor mutants displayed wild-type colony forming ability and colony size under permissive conditions (Fig. 1b). The colony-forming ability of all three suppressor-bearing strains was more than 10,000fold higher than the ftsZ84 parent strain under nonpermissive conditions (Fig. 1b). ftsZ84* suppressor colonies appeared to be slightly smaller than those of the wildtype strain, but were otherwise morphologically normal (Fig. 1b).

\section{Whole genome sequencing of ftsZ84 revealed linked mutations in $\mathrm{fts} W$ and murE}

In the course of sequencing suppressor mutations, we identified 2 additional mutations located between ftsZ84 and the tet $R$ marker that differed from the MG1655 parent. These point mutations, in fts $W$ and murE respectively, were inseparable from the ftsZ84 mutation by $\mathrm{P} 1$ transduction. The tight linkage between all three genes (ftsZ84, fts W, and murE) suggests that these mutations are the consequence of the $\mathrm{N}$-nitrosoguanidine 


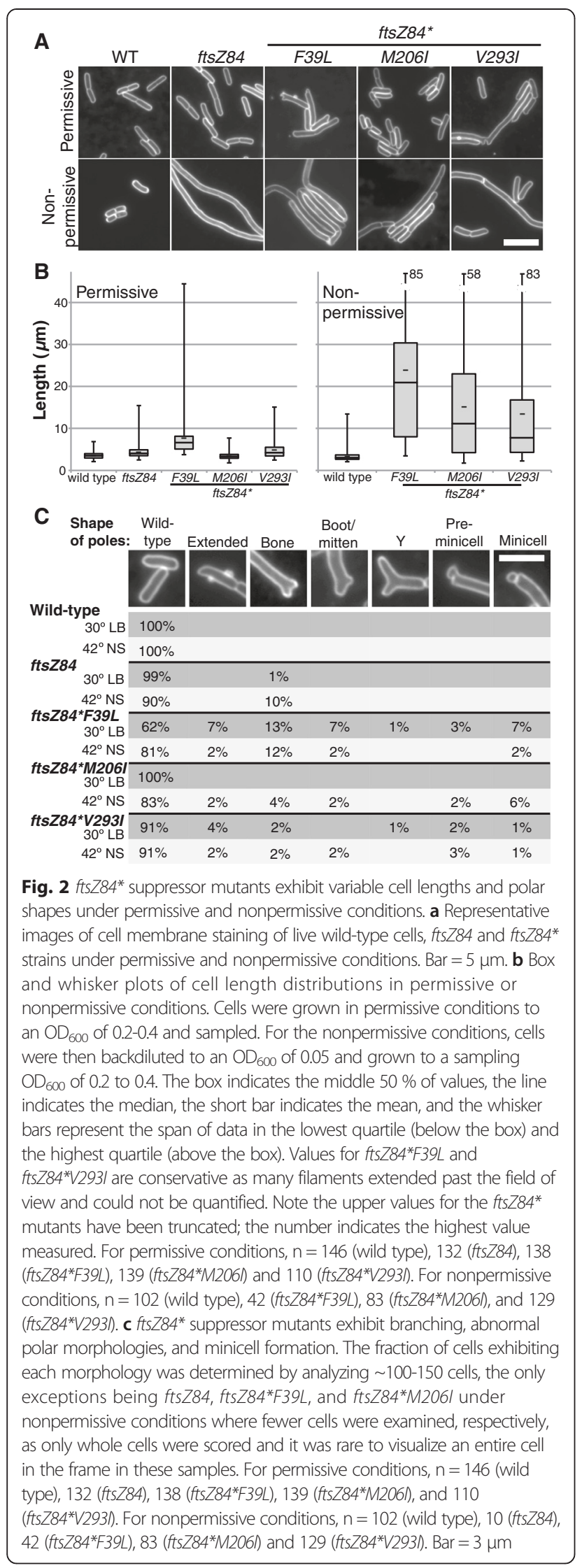

mutagenesis used to generate the original ftsZ84 (PAT84) strain $[12,19]$.

$\mathrm{FtsW}$ is an integral membrane protein that stabilizes the FtsZ ring and recruits FtsI (PBP3) to the division site $[2,20,21]$. The guanine to adenine mutation at residue 44 replaces a glycine with glutamic acid in the first transmembrane domain (G15E). Given its essential nature, we wondered if insertion of the charged glutamic acid residue in the first hydrophobic transmembrane domain of FtsW by virtue of the G15E mutation might reduce its potential to be accurately inserted in the membrane and contribute to the heat sensitivity of ftsZ84 cells. However, expression of wild-type $f t s W$ from a plasmid in the fts $Z 84$ background (HA223) did not complement the heat sensitivity of ftsZ84 cells, suggesting the fts W G15E mutation does not play a role in the conditional ftsZ84 phenotype (data not shown).

MurE is a cytoplasmic protein that catalyzes the addition of mesodiaminopimelate to peptidoglycan monomers [22]. The guanine to adenine mutation at position 1381 in $m u r E$ changes a valine to an isoleucine in a poorly conserved region 34 residues from the $\mathrm{C}$-terminus (V461I). In addition to the V461I mutation, there is also a synonymous mutation at base pair 1113, G1113A, which is in the wobble position and encodes for the correct amino acid (alanine) at position 371. Due to the conservative nature of the V461I mutation in MurE, we did not determine if exogenous murE expression suppressed the ftsZ84 phenotype.

\section{Intragenic suppressors grow more slowly than wild-type cells under nonpermissive conditions}

Despite being near wild type for colony-formation, all three intragenic suppressor mutants exhibited detectable reductions in growth rate in liquid medium under nonpermissive conditions as compared to wild-type cells (Table 1). Wild-type MG1655 E. coli had a mass doubling time of 26.2 minutes in no salt media at $42{ }^{\circ} \mathrm{C}$ and ftsZ84 cells grew at approximately the same rate, increasing exponentially under nonpermissive conditions for $\sim 4$ doubling periods before growth arrested. This increase in mass for several generations prior to a rapid growth arrest parallels what we have observed in $B$. subtilis cells subjected to a prolonged block in division (Fig. 1c, Table 1) [23]. Consistent with their reduced colony size, all three intragenic suppressor strains exhibited reduced rates of mass increase under nonpermissive conditions. Of the three intragenic suppressors, ftsZ84*F39L exhibited the largest growth defect, taking more than twice the time of wild-type MG1655 cells to double in mass ( 57 minutes) (Fig. 1c, Table 1). ftsZ84*M206I and ftsZ84*V293I cells grew more robustly, albeit slower than their wild - type counterparts (mass doubling times $~ 34$ and 38 minutes, respectively) (Fig. 1c, Table 1). We 
Table 1 Doubling times of $\mathbf{f t s Z 8 4 *}$ strains. Strains were grown in permissive conditions $\left(\mathrm{LB}\right.$ at $30^{\circ} \mathrm{C}$ ) or nonpermissive conditions ( $\mathrm{LB}-\mathrm{no}$ salt at $42^{\circ} \mathrm{C}$ ) and doubling times during exponential phase were calculated

\begin{tabular}{lll}
\hline & $\begin{array}{l}\text { Permissive Doubling } \\
\text { Time }(\mathrm{min})\end{array}$ & $\begin{array}{l}\text { Nonpermissive Doubling } \\
\text { Time }(\mathrm{min})\end{array}$ \\
\hline WT & $49.4( \pm 5.2)$ & $26.2( \pm 2.8)$ \\
$\mathrm{ftsZ84}$ & $47.6( \pm 7.1)$ & na \\
$\mathrm{ftsZ84*F39L}$ & $51.5( \pm 3.1)$ & $57.3( \pm 2.1)$ \\
$\mathrm{ftsZ84*M2061}$ & $51.4( \pm 2.7)$ & $33.6( \pm 3.2)$ \\
$\mathrm{ftsZ84*V2931}$ & $49.0( \pm 4.1)$ & $38.4( \pm 3.3)$ \\
\hline
\end{tabular}

observed no detectable difference in mass doubling time between strains under permissive conditions (Table 1).

Importantly, all three intragenic suppressors had little impact on the intracellular concentration of FtsZ84 (Fig. 1d). On quantitative immunblots probed with antisera generated against $E$. coli FtsZ (the gift of David Weiss), FtsZ84 levels were $\sim 90 \%$ of wild-type levels and the levels of all mutants were similar to FtsZ84 or wild-type levels (FtsZ84*F39L was $84 \%$ of wild-type, FtsZ84*M206I was $101 \%$ of wild-type levels and FtsZ84*V293I was $90 \%$ of wild-type levels) (Fig. 1d). These data indicate that all three mutations suppress ftsZ84 associated heat sensitivity by altering FtsZ-ring assembly or maturation dynamics rather than elevating FtsZ levels, which has previously been shown to suppress ftsZ84 [24, 25].

\section{Intragenic suppressors partially rescue ftsZ84 filamentation and FtsZ ring formation under nonpermissive conditions}

While the intragenic suppressor mutations restored growth and colony formation to ftsZ84 mutants under nonpermissive conditions, all three ftsZ84* strains exhibited increases in cell length, consistent with retaining a partial defect in cell division. Under nonpermissive conditions (LB-no salt medium at $42{ }^{\circ} \mathrm{C}$ ), wild-type MG1655 E. coli averaged $\sim 3.4 \mu \mathrm{m}$ in length (range $=2$ to $13 \mu \mathrm{m}$ ). ftsZ84 cells were extensively filamentous, as previously reported (Fig. 2) [12]. (Precise length could not be determined, as the majority of ftsZ84 filamentous cells extended beyond the field of view.) Of the ftsZ84* mutants, ftsZ84*F39L mutants exhibited the most filamentous phenotype under nonpermissive conditions with an average length of $24 \mu \mathrm{m}$ (range $=3.5$ to $85 \mu \mathrm{m}$ ) (Fig. 2). (Because very long filaments extended out of the field of view, the calculated average is likely to be a significant underestimate.) Although $f t s Z 84 * M 206 I$ and ftsZ84*V293I mutants rescued the cell division phenotype to a greater extent than ftsZ84:F39L, both strains exhibited increases in cell size with average lengths of $\sim 15 \mu \mathrm{m}$ (range 1.7 to $58 \mu \mathrm{m}$ ) and $13 \mu \mathrm{m}$ (range 2.2 to $83 \mu \mathrm{m}$ ) respectively (Fig. 2).
As expected based on their ability to suppress ftsZ84 associated lethality, all three intragenic suppressors restored FtsZ ring formation to ftsZ84* cells cultured under nonpermissive conditions. In contrast to ftsZ84 cells which had no observable rings (length/ring (L/R) ratio $>400 \mu \mathrm{m}$ ), ftsZ84*M206I and ftsZ84*V293I exhibited L/R ratios of $48 \mu \mathrm{m}$ and $23 \mu \mathrm{m}$ respectively (Fig. $3 \mathrm{~b}$ ). Consistent with its highly filamentous nature ftsZ84*F39L mutants had very few FtsZ rings under nonpermissive conditions $(L / R$ ratio $\sim 100 \mu \mathrm{m})($ Fig. $3 \mathrm{~b})$. For reference, wild-type cells exhibited an $\mathrm{L} / \mathrm{R}$ ratio of $6.1 \mu \mathrm{m}$ in LB-no salt at $42{ }^{\circ} \mathrm{C}$ in (Fig. 3).

\section{ftsZ84*F39L and ftsZ84*V293I mutants exhibit increases in cell length under permissive conditions}

Intriguingly, two of the fts $Z 84 \%$ intragenic suppressor mutants, ftsZ84*F39L and ftsZ84*V293I, exhibited significant increases in cell length under permissive conditions, suggesting persistent defects in FtsZ assembly. Under permissive conditions, wild-type cells averaged $\sim 3.5 \mu \mathrm{m}$ in length with a cell length distribution between 2.1 and $6.8 \mu \mathrm{m}$, in agreement with previous reports [26] (Fig. 2). Both the ftsZ84 parent strain and ftsZ84\%M206I exhibited near wild-type lengths and were essentially wild type with regard to size under permissive conditions, consistent with near normal rates of division $(\sim 4.3 \mu \mathrm{m}$, distribution 2.5 to $15 \mu \mathrm{m}$ and $\sim 3.4 \mu \mathrm{m}$, distribution 1.8 to $7.7 \mu \mathrm{m}$ respectively) (Fig. 2). In contrast to both the ftsZ84 parent and ftsZ84*M206I, under permissive conditions ftsZ84*F39L and ftsZ84*V293I cells were significantly longer than wild type, suggesting a persistent defect in division. ftsZ84*F39L mutants had a wide distribution of lengths (3.8 to $44 \mu \mathrm{m}$ ) with an average of $7.7 \mu \mathrm{m}$ (Fig. 2). While somewhat less filamentous, ftsZ84*V293I cells were $\sim 40 \%$ longer than wild-type cells and exhibited a size distribution similar to the ftsZ84 parent (2.4 to $15 \mu \mathrm{m})$ under permissive conditions (Fig. 2).

The $L / R$ ratios of the suppressor strains did not always track with their length distributions under permissive conditions, suggesting that at least a subset of the ftsZ84* mutants are defective in aspects of division subsequent to FtsZ ring formation. Wild-type cells, ftsZ84 mutants, and ftsZ84*M206I cells exhibited $\mathrm{L} / \mathrm{R}$ ratios of $\sim 6.5 \mu \mathrm{m}, \sim 7.2 \mu \mathrm{m}$, and $\sim 7.8 \mu \mathrm{m}$, respectively (Fig. 3a). While ftsZ84*F39L cells exhibited an elevated $\mathrm{L} / \mathrm{R}$ ratio of $13 \mu \mathrm{m}$ under permissive conditions, consistent with their filamentous nature, ftsZ84*V293I mutants exhibited a wild-type $L / R$ ratio $(\sim 6.3 \mu \mathrm{m})$ under permissive conditions despite their large distribution of cell sizes (Fig. 2, 3a). This discrepancy between cell length distribution and $L / R$ ratio suggests that while FtsZ84*V293I is capable of assembly under permissive conditions, FtsZ84*V293I rings are impaired for function. 


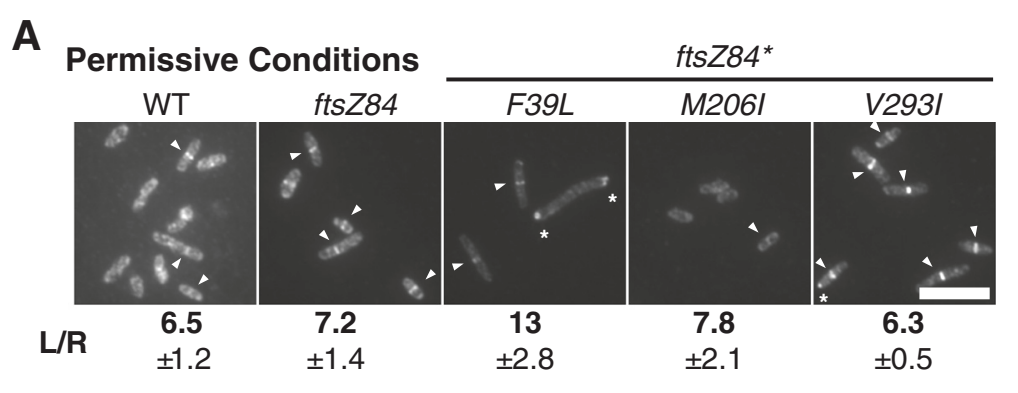

B Nonpermissive Conditions

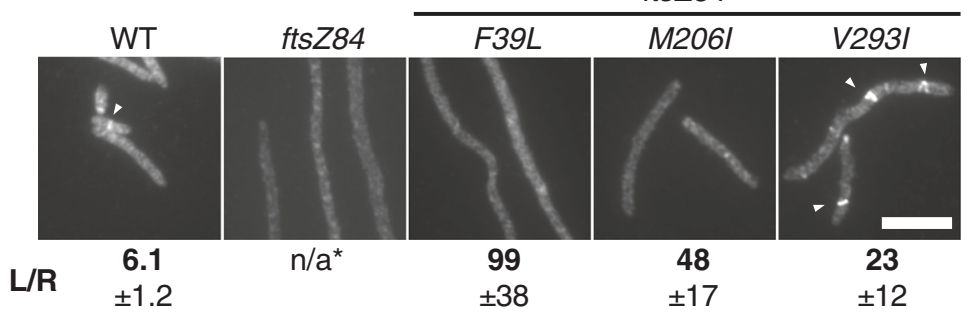

Fig. $3 \mathrm{fts} Z 84^{*}$ suppressor mutations restore FtsZ ring formation to varying degrees under nonpermissive conditions. Representative images of cells grown under (a) permissive conditions or (b) nonpermissive conditions and labeled for cell wall and FtsZ. Arrowheads indicate FtsZ rings. Bar $=5 \mu \mathrm{m}$. The length per ring $(L / R)$ ratio was calculated by dividing the total length of all cells by the number of Fts $Z$ rings counted. Average and standard deviation of 3 biological replicates is shown below each image

\section{ftsZ84* suppressor mutants exhibit abnormal polar morphologies}

Work from the Young laboratory indicates that defects in FtsZ ring orientation and/or structure lead to the formation of polar extensions, minicells, and Y-shaped branched cells - the result of aberrant insertion of inert peptidoglycan (normally only inserted at cell poles) [27]. Consistent with defects in FtsZ localization and/or cytokinetic ring integrity, we occasionally observed abnormal polar morphology and cell branching in ftsZ84* mutants. ftsZ84*F39L mutants exhibited the most severe polar phenotypes with $\sim 40 \%$ and $\sim 20 \%$ of cells displaying abnormal polar phenotypes (branching, polar extensions, and minicells) under permissive and nonpermissive conditions respectively (Fig. 2c). The other mutants, ftsZ84*M206I and ftsZ84*V293I exhibited wild-type or near wild-type FtsZ localization under permissive conditions (Fig. 2c). However, in both cases we observed a slight increase in polar extensions and/or minicells under nonpermissive conditions (Fig. 2c).

Intragenic suppressor mutations increase FtsZ84mediated GTP hydrolysis at standard GTP concentrations Based on the observation that all three fts $Z 84^{*}$ alleles supported cytokinetic ring formation, we speculated that the intragenic suppressor mutations increase FtsZ84 subunit-subunit affinity - either directly or by enhancing interaction with GTP - to levels sufficient to permit assembly in vivo in the presence of stabilizing modulatory proteins but insufficient to allow assembly in vitro. To explore this possibility, we evaluated the ability of the FtsZ84\% mutants to hydrolyze GTP in vitro under conditions standardly employed to assess FtsZ function (pH 6.5, 1 mM GTP) [8, 10, 28]. Because two FtsZ monomers must interact to form the GTP hydrolysis site, GTPase activity is commonly employed as a proxy for FtsZ dimerization potential [8, 28, 29].

Consistent with increases in dimerization affinity, under standard conditions all three FtsZ84* proteins displayed an approximately 6-fold increase in GTPase activity relative to the FtsZ84 (G105S) single mutant. Under standard reaction conditions (50 $\mathrm{mM} \mathrm{MES,} \mathrm{pH} 6.5,50 \mathrm{mM} \mathrm{KCl}$, $2.5 \mathrm{mM} \mathrm{MgCl}_{2}, 1 \mathrm{mM}$ EGTA, $1 \mathrm{mM}$ GTP, $5 \mu \mathrm{M}$ FtsZ, $30{ }^{\circ} \mathrm{C}$ ), wild-type FtsZ GTPase activity was 7 GTP/FtsZ/ min and FtsZ84 was 0.47 GTP/FtsZ/min (Table 2). GTP hydrolysis rates for the three intragenic suppressors were $\sim 50 \%$ of wild-type FtsZ at $\sim 3 \mathrm{GTP} / \mathrm{FtsZ} /$ minute for FtsZ84*F39L, FtsZ84*M206I, and FtsZ84*V293I, respectively (Table 2). The enhanced GTPase activity exhibited by all three FtsZ84* mutants is consistent with relatively robust dimerization potential.

\section{FtsZ84* mutants restore GTP binding but not catalytic rate to FtsZ84}

The position of the intragenic suppressor mutations outside FtsZ's GTP binding pocket makes it unlikely that the interaction between FtsZ84* monomers and GTP is directly impacted. At the same time, the substantial increase in GTP hydrolysis exhibited by the FtsZ84* mutants suggests that increased subunit-subunit interaction 
Table 2 FtsZ GTPase turnover rates. GTPase assays were carried out in identical conditions to the standard conditions used for light scattering and EM (50 mM MES, pH 6.5, $50 \mathrm{mM} \mathrm{KCl}$, $2.5 \mathrm{mM} \mathrm{MgCh}, 1 \mathrm{nM}$ EGTA, $1 \mathrm{mM} \mathrm{GTP}, 30^{\circ} \mathrm{C}$ ). Rates were calculated as GTP consumed/FtsZ subunit/min

\begin{tabular}{llc}
\hline FtsZ species & GTP/FtsZ/min & Std. dev. \\
\hline FtsZ (wild type) & 7 & \pm 2.28 \\
FtsZ84 (G105S) & 0.47 & \pm 0.05 \\
FtsZ84*F31 & 3.1 & \pm 0.58 \\
FtsZ84*M2061 & 3 & \pm 1.29 \\
FtsZ84*V2931 & 3 & \pm 1.06 \\
FtsZ*F39L & 0.9 & \pm 0.19 \\
FtsZ*M2061 & 6 & \pm 1.50 \\
FtsZ*V2931 & 3 & \pm 1.16 \\
\hline
\end{tabular}

and/or conformational changes compensate for the GTP binding defect associated with the G105S mutation. To investigate this possibility, we generated Michaelis-Menten curves for FtsZ, FtsZ84, and the three FtsZ84* mutants by varying GTP concentration while maintaining FtsZ concentration at $5 \mu \mathrm{m}$ under our standard buffer conditions (50 mM MES, pH 6.5, $50 \mathrm{mM} \mathrm{KCl,} 2.5 \mathrm{mM} \mathrm{MgCl} 2,1 \mathrm{nM}$ EGTA, 0.25-4.0 mM GTP, $22{ }^{\circ} \mathrm{C}$ ). At higher concentrations of GTP ( $>4 \mathrm{mM}$ for wild-type FtsZ) we observed substrate inhibition and these points were excluded from out data set. The GTPase activity of FtsZ has previously been shown to follow Michaelis-Menten kinetics by two groups using different buffer conditions [30, 31].

To determine $K_{m}$ and $V_{\max }$ for all five FtsZ variants, we fit the GTPase activity data to a Hill-modified Michaelis-Menten equation, as that provided the best fit for our data (Fig. 4). $K_{m}$ provides an estimate of the disassociation constant $\left(K_{\mathrm{d}}\right)$ which correlates inversely with the affinity of the interaction between FtsZ and GTP. $V_{\max }$ is a reflection of the maximal rate of catalysis. Although interpretation is not as straightforward as it might be due to FtsZ's nature as a polymerizing enzyme, $n$ is a measure of cooperative binding $(n<1$ indicates cooperitivity).

$\mathrm{K}_{\mathrm{m}}$ data suggest that the amino acid substitutions in the FtsZ84* mutants compensate for the impact of the G105S mutation by enhancing interactions between FtsZ and GTP. Wild-type FtsZ had a $\mathrm{K}_{\mathrm{m}}$ of $1.3 \mathrm{mM}$, similar to the value obtained by Salvarelli et al. $(0.3 \mathrm{mM})$ but much higher than the $\mathrm{Km}$ of $82 \mu \mathrm{M}$ reported by Sossong et al. [30, 31]. Differences between our data and that of Sossong et al. can potentially be attributed to the different reaction conditions used in their study [30, 31]. In contrast to wild-type FtsZ, FtsZ84 exhibited a $K_{m}$ of $3.6 \mathrm{mM}$ (Table 3). The 3-fold increase between the $K_{\mathrm{m}}$ of wild-type FtsZ and the $\mathrm{K}_{\mathrm{m}}$ of FtsZ84 is consistent with previous work indicating that FtsZ84 is defective in GTP binding $[14,15]$. All three FtsZ84* mutants exhibited $K_{m}$ values approximately seven-fold less than FtsZ84, supporting the idea that the secondary mutations enhance interactions between FtsZ84 and GTP (Table 3). FtsZ84*F39L, FtsZ84*M206I and FtsZ84*V293I displayed $\mathrm{K}_{\mathrm{m}}$ values ranging from 0.5-0.6 $\mathrm{mM}$ (Table 3).

In contrast to $\mathrm{K}_{\mathrm{m}}$, the maximum catalytic rates $\left(\mathrm{V}_{\max }\right)$ of FtsZ84 and the three intragenic suppressor mutants were significantly lower than wild-type FtsZ, suggesting the original G105S mutation interferes with catalysis independent of GTP binding. Wild-type FtsZ exhibited a $\mathrm{V}_{\max }$ of $17 \mathrm{GTP} / \mathrm{FtsZ} / \mathrm{min}$ (Salvarelli et al. reported a $\mathrm{V}_{\max }$ of 6.0 molecules GTP/ FtsZ/min and Sossong et al. reported 19.7 GTP/FtsZ/min [30, 31]). The $\mathrm{V}_{\max }$ of FtsZ84 was reduced $\sim 30 \%$ to $12 \mathrm{GTP} / \mathrm{FtsZ} / \mathrm{min}$ (Table 3). The $\mathrm{V}_{\max }$ values of all three FtsZ84* mutants were notably lower than FtsZ84, ranging from 5-6 molecules GTP/ molecule FtsZ/min (Table 3). The lower $\mathrm{V}_{\max }$ of the three intragenic suppressors may be a consequence of enhanced subunit-subunit interaction, which could interfere with nucleotide exchange and/or changes in the conformation of the active site that prevent optimal GTP hydrolysis.

Consistent with models for cooperative assembly in which GTP binding by one subunit leads to substrate binding effects on neighboring subunits, wild type FtsZ, as well as FtsZ84 and FtsZ84* V293I, exhibit positive cooperativity $(n>1)$ (Table 4$)$ [32]. FtsZ84*F39L and FtsZ84*M206I on the other hand do not exhibit the same degree of positive cooperativity $(n=\sim 1)$.

\section{FtsZ84*F39L acts synergistically with FtsZ84 (G105S) to restore GTPase activity}

To gain an understanding of the impact of the three intragenic suppressor mutations on FtsZ assembly independent of FtsZ84 (G105S), we generated FtsZ constructs that are wild type at position 105, but harbor the individual suppressor mutations (FtsZ*) (Fig. 1b). Intriguingly, all three intragenic suppressor mutations reduced FtsZ mediated GTP hydrolysis independent of FtsZ84 (G105S) in our standard reaction conditions $(5 \mu \mathrm{M}$ FtsZ, $1 \mathrm{mM}$ GTP, $30{ }^{\circ} \mathrm{C}$ ). FtsZ*F39L, in particular, reduced GTPase activity $\sim 10$-fold, indicating that the F39L mutation functions synergistically with the G015S mutation to restore GTPase activity. FtsZ*F39L hydrolyzed only 0.9 GTP/FtsZ/min, a rate comparable to FtsZ84 (0.47 GTP/FtsZ/min) and well below the FtsZ84*F39L hydrolysis rate of $\sim 3 \mathrm{GTP} / \mathrm{FtsZ} / \mathrm{min}$ (Table 2). FtsZ*M206I exhibited a modest $\sim 20 \%$ reduction in GTPase activity to 6 GTP/FtsZ/min and FtsZ"V293I had an $\sim 65 \%$ reduction to $3 \mathrm{GTP} / \mathrm{FtsZ} / \mathrm{min}$ (Table 2).

\section{Intragenic suppressor mutations fail to restore FtsZ84 polymerization in vitro}

A primary question was whether the enhanced subunitsubunit interaction, as evidenced by increased GTPase 

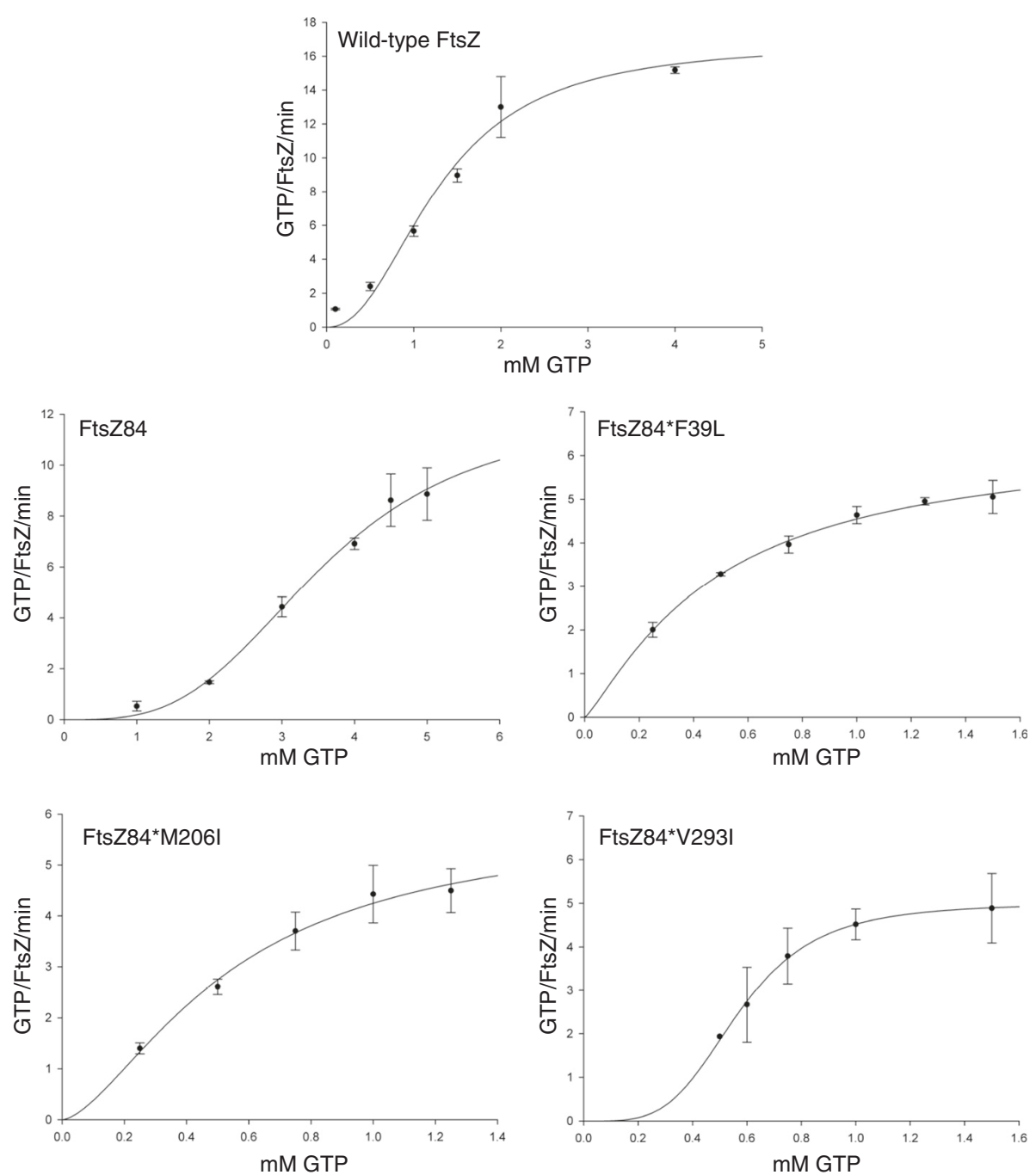

Fig. 4 Steady state kinetic analysis of FtsZ, FtsZ84, and FtsZ84* proteins. GTPase assays were carried out as described in materials and methods ( $50 \mathrm{mM}$ MES, pH 6.5, $50 \mathrm{mM} \mathrm{KCl}, 2.5 \mathrm{mM} \mathrm{MgCl} 2,1 \mathrm{mM}$ EGTA). FtsZ concentration was kept constant at $5 \mu \mathrm{M}$ and GTP concentration were varied from $0.1 \mu \mathrm{M}$ to $5 \mu \mathrm{M}$ (different ranges were used depending on the FtsZ mutant analyzed). The best fit for our data was obtained using a Hill-modified Michaelis-Menten equation using Sigma Plot software. $K_{m}$ and $V_{\max }$ values are listed in Table 3

activity, was sufficient to support FtsZ84: polymer formation in vitro. To investigate this possibility, we examined the assembly dynamics of purified FtsZ84* proteins using two standard FtsZ assays: $90^{\circ}$ angle light scattering and electron microscopy (EM). Based on the principle that polymers and polymer bundles will scatter more light than smaller structures such as FtsZ monomers, $90^{\circ}$ angle light scattering is a standard assay for FtsZ assembly in vitro [10, 33-35]. A limitation of light scattering is its inability to distinguish between a large number of relatively small structures and a few very large structures. Electron microscopy (EM), in contrast, although not quantifiable, allows direct visualization of FtsZ ultrastructures and can distinguish even small polymers [10, 34, 35]. Together, these two approaches provide both a quantitative and a qualitative measure of FtsZ's in vitro polymerization potential.

Despite their ability to support cytokinetic ring formation in vivo under nonpermissive conditions, all three intragenic suppressor mutations failed to restore FtsZ84 polymerization in vitro. As previously reported, in standard buffer conditions (50 mM MES, $\mathrm{pH} 6.5,50 \mathrm{mM} \mathrm{KCl}$, $2.5 \mathrm{mM} \mathrm{MgCl}_{2}, 1 \mathrm{mM}$ EGTA) the addition of GTP stimulates assembly of wild-type E. coli FtsZ into singlestranded protofilaments readily visible by EM (Fig. 5d) $[4,36]$. In contrast, FtsZ84, FtsZ84*F39L, FtsZ84*M206I, and FtsZ84*V293I all failed to assemble in vitro following the addition of GTP (Fig. 5). The light scattering signal remained near baseline and even decreased slightly for all 3 FtsZ84* mutant proteins and no FtsZ polymers 
Table 3 Steady state kinetic analysis of FtsZ, FtsZ84 and FtsZ84*. GTPase assays were carried out in identical conditions as the standard GTPase assay (Table 2) except GTP concentration was varied and the reactions were performed room temperature (50 mM MES, pH 6.5, $50 \mathrm{mM} \mathrm{KCl}, 2.5 \mathrm{mM} \mathrm{MgCl} 2,1$ mM EGTA, 0.25-4.0 mM GTP, $22^{\circ} \mathrm{C}$ ). Km and Vmax values were determined by fitting the data points to a Hill-modified Michaelis-Menten equation (see Fig.4 for the data and curve fitting of each protein)

\begin{tabular}{lccc}
\hline FtsZ species & $\mathrm{K}_{\mathrm{m}}(\mathrm{mM})$ & $\mathrm{V}_{\max }$ (GTP/FtsZ/min) & $\mathrm{n}$ \\
\hline FtsZ (wild type) & $1.3 \pm 0.18$ & $17 \pm 1.9$ & $2.2 \pm 0.56$ \\
FtsZ84 (G105S) & $3.6 \pm 0.79$ & $12 \pm 3.6$ & $3 \pm 1.1$ \\
FtsZ84*F39L & $0.5 \pm 0.14$ & $6 \pm 1.1$ & $1.2 \pm 0.32$ \\
FtsZ84*M2061 & $0.5 \pm 0.26$ & $6 \pm 1.9$ & $1.5 \pm 0.70$ \\
FtsZ84*V2931 & $0.57 \pm 0.069$ & $5.0 \pm 0.83$ & $4 \pm 2.7$ \\
\hline
\end{tabular}

Table 4 Strains and plasmids used in this study

\begin{tabular}{|c|c|c|}
\hline Strain & Genotype & Reference/Source \\
\hline MG1655 (PL2036) & F- lambda-ilvG-rfb-50 rph-1 & \\
\hline DRC14 & MC4100 ftsZ84(ts) leu::Tn10 & John Beckwith \\
\hline ftsZ84 (PL2452) & MG1655, leu82::Tn10 ftsZ84(ts) & \\
\hline ftsZ84*F39L (HA209) & MG1655, leu82::Tn10 ftsZ84*F39L & This study \\
\hline ftsZ84 *M206l (HA211) & MG1655, leu82::Tn10 ftsZ84 *M206l & This study \\
\hline ftsZ84 *V293I (HA213) & MG1655, leu82::Tn10 ftsZ84 *V2931 & This study \\
\hline HA223 & MG1655, leu82::Tn10 ftsZ84(ts), pBAD-ftsW & This study \\
\hline zapA (HA235) & MG1655, zapA::kan & This study \\
\hline ftsZ84, zapA (HA241) & ftsZ84, zapA::kan & This study \\
\hline ftsZ84*F39L, zapA (HA267) & ftsZ84*F39L, zapA::kan & This study \\
\hline ftsZ84*M2061, zapA (HA245) & ftsZ84*M206l, zapA::kan & This study \\
\hline ftsZ84*V2931, zapA (HA251) & ftsZ84*V293I, zapA::kan & This study \\
\hline HA255 & MG1655, pKG110-zapA & This study \\
\hline HA257 & ftsZ84, pKG110-zapA & This study \\
\hline CW206 & MG1655, pQE80-H6-zapA & This study \\
\hline HA364 & ftsZ84, pQE80-H6-ZapA & This study \\
\hline Plasmid/Strain harboring plasmid & Genotype & Reference \\
\hline pPJ2 (PL3142) & pET21b(+)-ftsZ & Buske and Levin, 2012 \\
\hline HA146 & pET21b(+)-ftsZ84 & This study \\
\hline HA149 & pET21b(+) -ftsZ84*F39L & This study \\
\hline HA147 & pET21b(+)-ftsZ84*M206l & This study \\
\hline HA142 & pET21b(+)-ftsZ84*V2931 & This study \\
\hline HA165 & $p E T 21 b(+)-f t s Z^{*} F 39 L$ & This study \\
\hline HA157 & pET21b(+)-ftsZ*M206l & This study \\
\hline HA166 & $p E T 21 b(+)-f t s Z^{*} V 2931$ & This study \\
\hline PL3463 & pBAD24-ftsW & This study \\
\hline HA255 & pKG110-zapA & Haeusser et al., 2014 \\
\hline HA364 & pQE80-H6-zapA & Dajkovic et al., 2001 \\
\hline
\end{tabular}

were evident in EM images (Fig. 5a,d). Notably, the appearance of FtsZ84 and FtsZ84* assembly reactions by EM is strikingly similar, suggesting that any differences in assembly that might be present are too subtle to visualize, even using this high-resolution technique. We should note that a more sensitive technique such as dynamic light scattering might reveal subtle differences in in vitro assembly that more standard approaches are unable to detect.

To determine if the defect in assembly of FtsZ84 and FtsZ84* was due, at least in part, to loss of lateral interaction potential or if it was primary due a deficiency in longitudinal subunit interactions, we increased the concentration of magnesium from $2.5 \mathrm{mM}$ to $10 \mathrm{mM}$ to stimulate lateral interactions between FtsZ protofilaments [33]. While the mechanism is likely different - charge shielding versus cross-linking-in vitro the addition of divalent cations largely mimics the impact of FtsZ-modulatory 

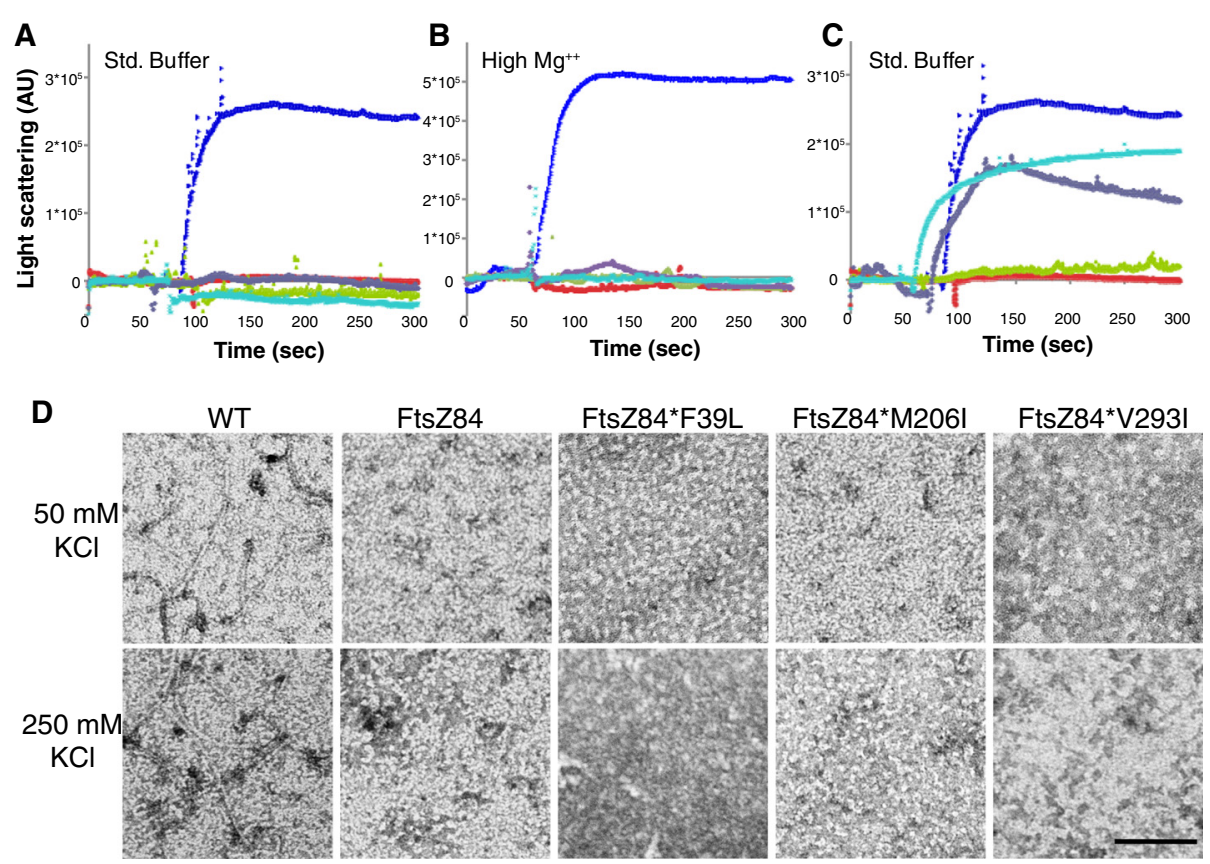

Fig. 5 fts $Z 84^{*}$ suppressor mutations do not restore assembly in vitro. a-b $90^{\circ}$ angle light scattering data from FtsZ (blue), FtsZ84 (red), FtsZ84*F39L (green), FtsZ84*M206l (purple), and FtsZ84*V2931 (aqua) a 50 mM MES, pH 6.5, 50 mM KCl, 2.5 mM MgCl 1 mM EGTA or b 50 mM MES, pH 6.5, 50 mM $\mathrm{KCl}, 10 \mathrm{mM} \mathrm{MgCl} 2,1 \mathrm{mM}$ EGTA (methods). After establishing baseline, assembly was initiated by the addition of $1 \mathrm{mM}$ GTP. c $90^{\circ}$ angle light scattering data from suppressor mutations alone (in the absence of ftsZ84(G105S)). FtsZ (blue), FtsZ84 (red), FtsZ*F39L (green), FtsZ*M206I (purple), and FtsZ*V2931 (aqua) Reaction conditions same as in (a). d Electron micrographs of FtsZ assembled in $50 \mathrm{mM} \mathrm{KCl}$ or $250 \mathrm{mM} \mathrm{KCl}(50 \mathrm{mM} \mathrm{MES}, \mathrm{pH} 6.5,50 \mathrm{mM} \mathrm{KCl}$, $2.5 \mathrm{mM} \mathrm{MgCl}_{2}, 1 \mathrm{mM}$ EGTA) $\mathrm{Bar}=100 \mathrm{~nm}$

proteins that enhance lateral interactions to bundle protofilaments [33, 37]. Consistent with a defect in longitudinal interactions, the addition of $10 \mathrm{mM}\left[\mathrm{Mg}^{++}\right]$had no impact on assembly of FtsZ84 or the three FtsZ84* mutant proteins in light scattering assays (Fig. 5). Increasing the potassium ion levels from $50 \mathrm{mM}$ to $250 \mathrm{mM}$, which has been reported to stimulate assembly of wild - type FtsZ proposedly through increasing stability of the GTP-bound FtsZ dimer [33, 38], also had no detectable impact on assembly of mutant FtsZ protein (Fig. 5d). Although $\mathrm{pH}$ has been reported to impact FtsZ, assembly, we elected not to vary $\mathrm{pH}$, as the conditions we employed ( $\mathrm{pH}$ 6.5) are the most permissive of the buffer conditions standardly employed for FtsZ analysis [2].

Light scattering indicates that the impact of the F39L, M206I and V293I mutations on assembly of otherwise wild-type FtsZ roughly parallels their impact on GTP hydrolysis. Under the standard reaction conditions described above, FtsZ*F39L protein assembled to levels only slightly above baseline, consistent with its $\sim 10$ fold reduction in GTP hydrolysis, while FtsZ*M206I and FtsZ*V293I assembled to levels $\sim 65 \%$ of wild type, consistent with their more robust GTPase activity (Fig. 5c).
Intragenic suppressors of ftsZ84 rely on other components of the cell division machinery for growth and division under nonpermissive conditions

Based on their inability to form stable protofilaments in vitro, we speculated that the all three FtsZ84*s as well as their FtsZ84 parent protein were dependent on modulatory proteins. One candidate protein ZapA has previously been shown to stabilize FtsZ protofilaments in vitro and in vivo $[17,39]$. To test if ZapA is helping stabilize FtsZ84* under nonpermissive conditions, we examined the impact of deleting zapA on the viability of ftsZ84 and ftsZ84* mutants under both permissive and nonpermissive conditions.

Although loss of zapA had no impact on colony forming ability under permissive conditions, zapA was required for full ftsZ84* mutant viability under nonpermissive conditions (LB no salt at $42{ }^{\circ} \mathrm{C}$ ) (Fig. 6). Under nonpermissive conditions, the zapA deletion reduced the colony forming ability of ftsZ84*F39L cells $\sim 1,000-10,000$-fold and the colony forming ability of ftsZ84*M206I and the ftsZ84*V293I 100-fold (Fig. 6b).

Since ZapA has been previously been reported to stabilize FtsZ protofilaments in vitro [17], we next tested whether overexpressing zapA would restore colony-forming ability to ftsZ84 cells under semi-permissive and nonpermissive 


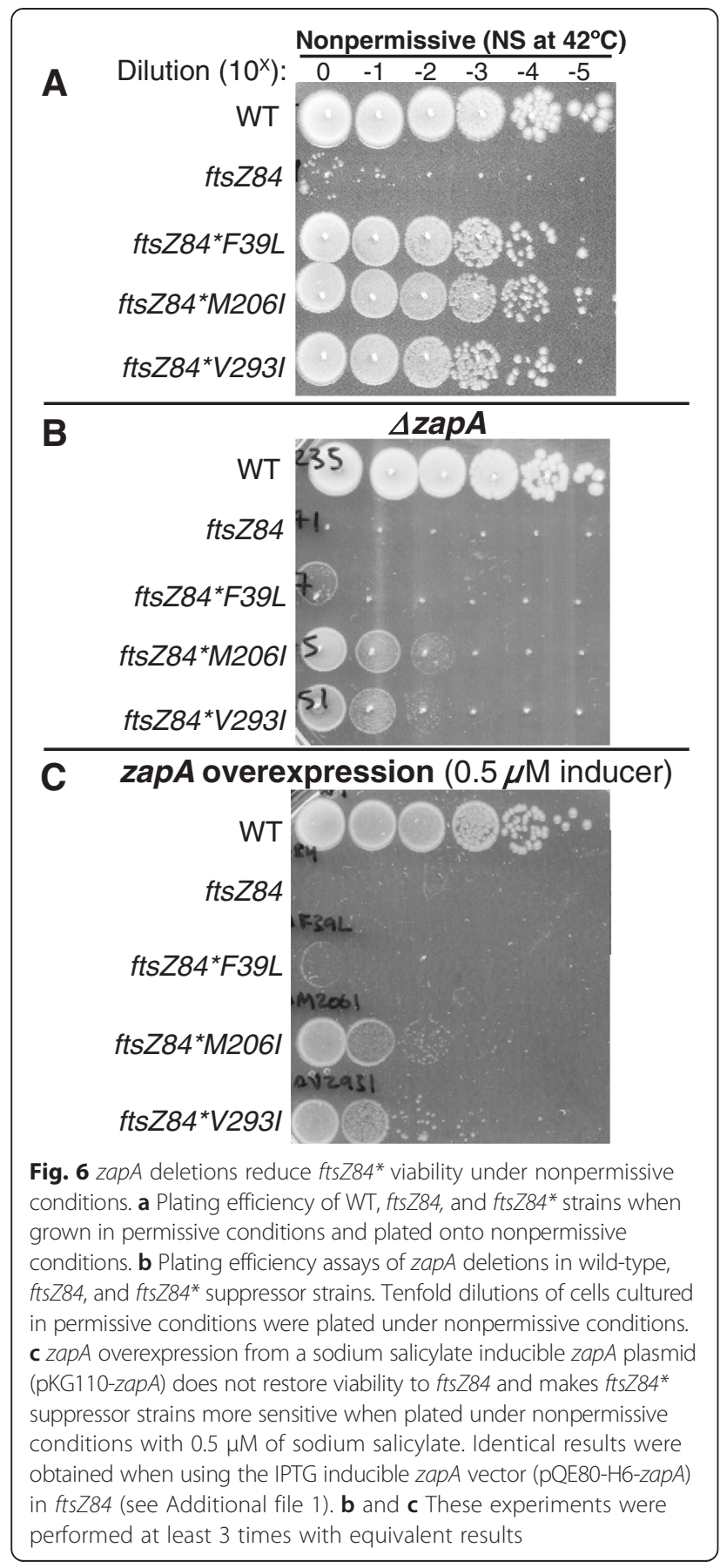

conditions. Surprisingly, overexpression of zapA from two different inducible/repressible promoters (a sodium salicylate inducible construct, the gift of Bill Margolin, and an IPTG-inducible construct, the gift of Alex Dajkovic) failed to restore growth to ftsZ84 strains under nonpermissive conditions, suggesting that ZapA is not the sole determinant of FtsZ84* assembly potential in vivo (Fig. 6c, Additional file 1: Figure S1). In fact, zapA expression from a strong plasmid based promoter significantly reduced the ability of all three ftsZ84* mutations to suppress
ftsZ84 heat sensitivity (Fig. 6c). In particular, zapA overexpression reduced colony formation in ftsZ84*F39L by 10,000 -fold and ftsZ84*M206I and ftsZ84*V293I by 100fold under non-permissive conditions (Fig. 6c).

\section{Discussion}

Our data support a model in which disruption of interactions between FtsZ monomers at the longitudinal subunit interface is the underlying cause of ftsZ84 conditional lethality (Fig. 7). Wild-type FtsZ is optimized for GTP binding and hydrolysis (Fig. 7a). Like tubulin, GTP binding leads to a conformational change that increases subunit-subunit affinity and promotes dimerization and protofilament formation [2]. As indicated by the increased $K_{m}$ of FtsZ84 as compared to wild-type FtsZ, the (G105S) mutation in the GTP binding site interferes with GTP binding (Fig. 7b). Based on our finding that the FtsZ84* mutants restore GTP binding as estimated by $\mathrm{K}_{\mathrm{m}}$, we propose the intragenic suppressor mutations increase subunit-subunit affinity and promote nucleotide binding by trapping GTP between in the active site between FtsZ subunits (Fig. 7c).

FtsZ84's $\mathrm{V}_{\max }$ is approximately $70 \%$ that of wild type FtsZ (Table 3) suggesting that FtsZ84 not only has a reduced affinity for GTP, but is also defective for catalysis. The intragenic suppressor mutations further reduce the FtsZ's catalytic rate from to $\sim$ half the rate of FtsZ84. This reduction in $V_{\max }$ may be due to (1) a conformational change in the protein that alters GTP binding

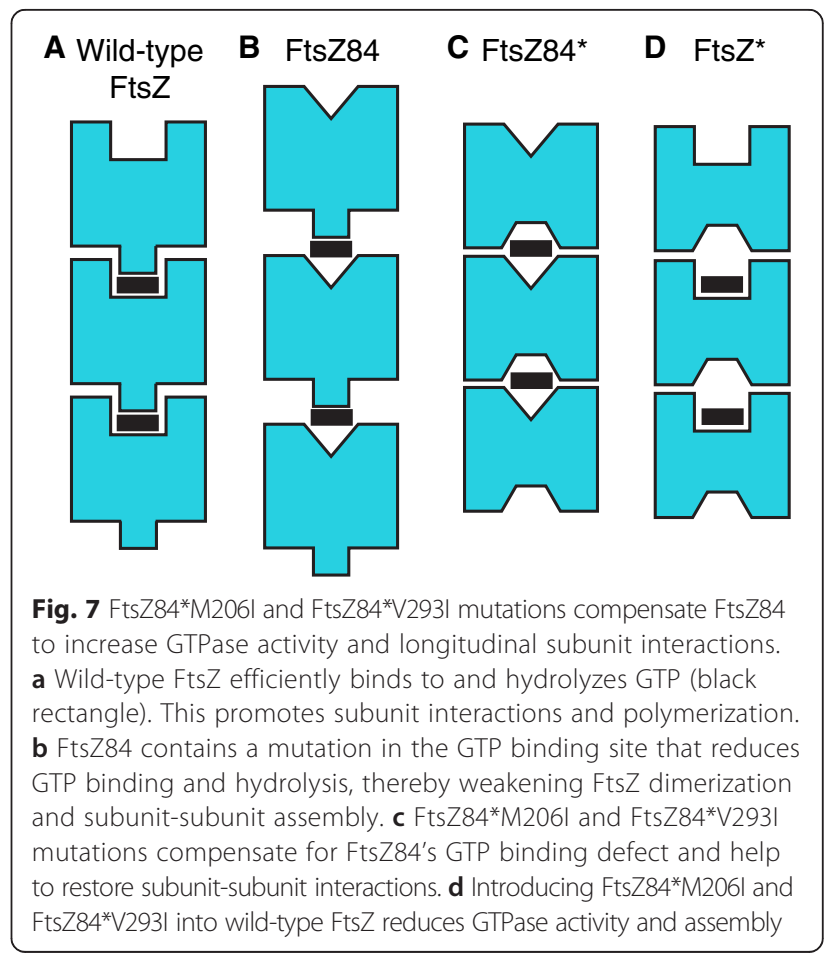


such that it is no longer in the optimal orientation for efficient hydrolysis and/or (2) an increased subunitsubunit interaction that promotes GTP retention at the subunit interface, limiting nucleotide exchange.

Enzyme kinetics suggest positive cooperativity with regard to GTP binding for wild-type FtsZ, FtsZ84 and FtsZ84*V293I $(n>1)$ (Table 4) consistent with the model for cooperative assembly suggested by Miraldi and Romberg [32]. However, the significance of this observation in unclear due to the challenge of assessing the value of $n$ for a polymerizing enzyme such as FtsZ that is reported to exchange nucleotide in the polymer itself [40, 41]. Moreover, neither FtsZ84*F39L nor FtsZ84*M206I exhibit positive cooperativity $(n=\sim 1$ for both). Further complicating interpretation is data from Salvarelli et al. suggesting that GTPase active sites are independent in FtsZ filaments [31]. Regardless, further work is needed to resolve the impact of nucleotide binding on FtsZ conformation and investigate the potential cooperative nature of FtsZ filaments.

The location of M206I in the T7 catalytic loop and V293I along the subunit-subunit interface suggests that both mutations directly enhance interactions between subunits and thus promote GTP retention at the active site (Fig. 7c). Consistent with this model, the M206I and V293I single mutants exhibited only moderate reductions in GTPase activity (Table 2, Fig. 7d). While exhibiting lower GTPase activity, FtsZ*M206I was not statistically different from wild type. FtsZ*V293I exhibited $\sim 2$-fold less GTPase activity relative to its wildtype parent. On the other hand, based on its location in the interior of the FtsZ core domain (Fig. 1), we favor the idea that the F39L defect suppresses ftsZ84 heat sensitivity by counteracting the impact of the G105S substitution through changes in the conformation of the GTP binding site. This model is consistent with FtsZ*F39L's significantly impaired GTPase activity in the absence of G105S.

We speculate that modulatory proteins that enhance FtsZ assembly are the underlying cause of the dichotomy in assembly potential exhibited by FtsZ84 and FtsZ84* in vitro and in vivo. FtsZ ring formation is generally thought to be a two-step process involving first the formation of transient, single stranded FtsZ polymers that are subsequently stabilized through lateral interactions mediated by a host of modulatory proteins [42]. The dynamic nature of the FtsZ ring means that maintenance of the ring similarly requires the both strong subunitsubunit interactions and subsequent stabilization by modulatory proteins [43]. Supporting the idea that defects in FtsZ assembly can be compensated for by intragenic mutations, the Margolin laboratory recently identified a mutation in E. coli FtsZ (L169W) that bypasses the requirement for the normally essential bundling protein
ZipA and confers resistance to defects in other components of the division machinery [44].

Applying this model to ftsZ84 cells under nonpermissive conditions, reduced subunit affinity would disrupt the early steps in FtsZ84 assembly limiting the formation of single stranded polymers and impacting downstream interactions with stabilizing modulatory proteins. Consistent with this model, shifting ftsZ84 mutants to nonpermissive conditions prevents the formation of new FtsZ rings and leads to the rapid disassembly of all extant FtsZ rings [45].

Based on our analysis of the FtsZ84* intragenic suppressor mutations, we propose the restoration of subunit-subunit affinity is sufficient to support the transient assembly of FtsZ84* polymers and subsequent interaction with modulatory proteins, ultimately permitting formation of a functional cytokinetic ring under nonpermissive conditions. In vitro, however, the absence of modulatory proteins precludes both FtsZ84 and FtsZ84* from forming polymers stable enough to detect by either $90^{\circ}$ angle light scattering or EM. Consistent with this model, the loss of ZapA, which stabilizes FtsZ protofilaments, severely reduces the viability of FtsZ84 and FtsZ84* mutants under nonpermissive conditions (Fig. 6b). Elucidation of the molecular mechanisms by which modulatory proteins promote FtsZ assembly and enhance the integrity of the FtsZ ring should clarify the apparent discrepancy between the assembly dynamics FtsZ84 and FtsZ84* in vitro and in vivo.

\section{Conclusions}

This study suggests a mechanism by which FtsZ84, a polymerizing enzyme with defective catalysis, gains function through increased substrate binding and enhanced subunit-subunit interactions. Although kinetic analysis clearly indicates a significant enhancement in substrate interaction (Fig. 5), this increase in $\mathrm{Km}$ is insufficient to restore assembly to FtsZ84* in vitro, although all three suppressor mutants supported assembly in vivo under both permissive and nonpermissive conditions. In theory, defects in assembly of other polymerizing enzymes might also be compensated for by increases in subunit affinity. While this increased binding is evident through enzyme kinetics, it does not restore assembly to FtsZ84* in common in vitro assembly reactions, although these proteins can assemble in vivo at both permissive and nonpermissive conditions.

By illuminating the disconnect between in vitro and in vivo assembly, this study highlights the value of pairing biochemistry with genetics and cytology in order to draw biologically meaningful conclusions. While light scattering and EM are invaluable for evaluating gross changes in FtsZ assembly, understanding FtsZ assembly dynamics in vivo will ultimately require the development of 
super resolution approaches for imaging of cytokinetic ring formation in real time as well as approaches to reconstitute FtsZ assembly in vitro in the context of its full complement of modulatory proteins.

\section{Methods}

General methods and strain construction

All E. coli strains are derivatives of MG1655 and are listed in Table 4.

The ftsZ84 allele from DRC14 (the gift of Debabrata RayChaudhuri) was moved into MG1655 via P1 phage transduction to create PL2452 (ftsZ84) [18, 46]. Cells were grown in permissive conditions in lysogeny-broth (LB) Miller (10 g/L tryptone, $5 \mathrm{~g} / \mathrm{L}$ yeast extract, $10 \mathrm{~g} / \mathrm{L}$ $\mathrm{NaCl})$ at $30{ }^{\circ} \mathrm{C}$ or nonpermissive conditions of $42{ }^{\circ} \mathrm{C}$ in LB-no salt media (10 g/L tryptone, $5 \mathrm{~g} / \mathrm{L}$ yeast extract) where indicated.

Alleles were moved between strains by P1 phage transduction [18]. Cloning, transformations and phage transduction were carried out under standard conditions [18, 47]. Ampicillin $(100 \mu \mathrm{g} / \mathrm{ml})$, tetracycline $(12.5 \mu \mathrm{g} / \mathrm{ml})$ and arabinose $(0.5 \%)$ were used as needed for maintenance of episomal DNA, selection of linked alleles following genetic exchange, and induction of gene expression, respectively.

\section{Whole genome sequencing}

The genomes of our ftsZ84 (PL2452) strain and the parental MG1655 strain (PL2036) were subjected to both Illumina and 454 sequencing courtesy of The Genome Institute at Washington University in St. Louis. Allelic differences between the wild-type MG1655 parental strain (PL2036) and ftsZ84 (PL2452) were confirmed by both Illumina and 454 sequencing.

Identification of intragenic suppressors of $\mathrm{ftsZ84}$

ftsZ84 cells were grown to mid-exponential phase $\left(\mathrm{OD}_{600}\right.$ 0.2-0.5) in permissive conditions $\left(30{ }^{\circ} \mathrm{C}, \mathrm{LB} 1 \%\right.$ $\mathrm{NaCl}) .1 \mathrm{ml}$ of cell culture was removed, washed once in LB - no salt media and plated onto LB-no salt plates at $42{ }^{\circ} \mathrm{C}$. Colonies were verified for temperature resistance and struck onto LB-no salt plates at $42{ }^{\circ} \mathrm{C}$. Suppressors were analyzed for linkage to ftsZ84 using P1 phage transduction [18] onto LB plates containing tetracycline and then transductants were patched onto nonpermissive conditions to determine whether temperature resistance was linked to $\mathrm{ftsZ84}$. The $\mathrm{ftsZ84}$ allele of linked mutations was amplified by polymerase chain reaction to determine if the suppressor mutation was intragenic. The ftsZ84 alleles containing intragenic suppressor mutations were moved by P1 transduction to our wild-type MG1655 background.

\section{EMS mutagenesis}

For ethyl methanesulfonate (EMS) mutagenesis, cells were grown to mid-exponential phase $\left(\mathrm{OD}_{600} 0.2-0.5\right)$ in permissive conditions. Cells were then treated with $25 \mu \mathrm{g} / \mathrm{ml}$ EMS for 45 minutes at 30 degrees (in LB-Miller $1 \%$ salt media) prior to 3 washes in LB-no salt media and plating on LB-no salt plates. Mutant verification was carried out as above.

\section{Growth rate determination}

Strains were grown under permissive conditions to midexponential phase $\left(\mathrm{OD}_{600}\right.$ 0.2-0.5). Cells were pelleted and washed once with LB-no salt media and back-diluted to a calculated $\mathrm{OD}_{600}$ of 0.025 in prewarmed $\left(42{ }^{\circ} \mathrm{C}\right)$ LB-no salt (nonpermissive conditions) or LB-1 \% NaCl at $30{ }^{\circ} \mathrm{C}$ (permissive conditions). Absorbance $\left(\mathrm{OD}_{600}\right)$ was measured every 30 minutes post back-dilution for 6 hours. The doubling time during exponential phase was calculated using www.doubling-time.com.

\section{Plating efficiency assay}

Cells were grown to mid-exponential phase $\left(\mathrm{OD}_{600} 0.2\right.$ 0.5) under permissive conditions. Cells were pelleted and washed once in LB-no salt media. Cells were resuspended in LB-no salt medium to a calculated $\mathrm{OD}_{600}$ of 0.2. $10 \mu \mathrm{l}$ of tenfold serial dilutions were spotted on LB agar and LB-no salt agar at $30{ }^{\circ} \mathrm{C}$ and $45^{\circ} \mathrm{C}$.

\section{Fluorescence microscopy}

For imaging of cells grown at $42{ }^{\circ} \mathrm{C}$ in LB-no salt media, cells were first cultured in $\mathrm{LB} 1 \% \mathrm{NaCl}$ at $30{ }^{\circ} \mathrm{C}$ to an $\mathrm{OD}_{600}$ of 0.2-0.5, washed once in LB-no salt media, and back-diluted to a calculated $\mathrm{OD}_{600}$ of 0.05 in prewarmed LB-no salt media. Cells were then cultured under nonpermissive conditions for 75 minutes ( 3 wild-type mass doublings).

\section{Live cell imaging}

For live cell imaging, the membrane stain FM4-64 was added to the cell sample at a final concentration of $1 \mu \mathrm{g} / \mathrm{ml}$ and incubated for 5 minutes. The cells were spotted on agarose pads containing $1 \%$ agarose in $1 \mathrm{X}$ phosphate buffered saline (PBS). Images were captured within 10 minutes of placing the cells on the slides. Cell length was calculated by measuring the distance of the cells from pole to pole. Only cells that were completely visible in the field were measured.

\section{Immunofluorescence microscopy}

Immunofluorescence microscopy was performed as previously described $[26,48]$. Briefly, cells were fixed through a glutaraldehyde-paraformaldehyde fixation. To prepare the fix, $6.25 \mu \mathrm{l}$ of $8 \%$ glutaraldehyde was added to $1 \mathrm{ml}$ of $16 \%$ paraformaldehyde on ice. Immediately before 
fixation, $100 \mu \mathrm{l}$ of the fix was added to $20 \mu \mathrm{l}$ of $1 \mathrm{M}$ $\mathrm{NaPO}_{4} \mathrm{pH}$ 7.4. $500 \mu \mathrm{l}$ of the cell culture was then added and the tubes were inverted. The samples were then incubated for 15 minutes at room temperature followed by 30 minutes on ice. Fixed cells were pelleted at $14,000 \mathrm{x}$ g in a microcentrifuge, washed 3 times with $1 \mathrm{~mL}$ of $1 \mathrm{X}$ PBS, resuspended in GTE $(50 \mathrm{mM}$ glucose, $25 \mathrm{mM}$ Tris, $10 \mathrm{mM}$ EDTA, $\mathrm{pH}$ 8.0) to a final $\mathrm{OD}_{600}$ of $\sim 0.2$ and stored at $4{ }^{\circ} \mathrm{C}$. To stain cells for immunofluorescence, samples were affixed to poly-l-lysine coated slides and then treated with $2 \mu \mathrm{g} / \mathrm{ml}$ lysozyme for 2 minutes to weaken the cell wall. Staining was as follows: cells were blocked with $2 \%$ bovine serum albumin (BSA) for 15 minutes, treated with 1:500 rabbit anti FtsZ antibody in $2 \% \mathrm{BSA}$ at $37^{\circ} \mathrm{C}$ for 30 minutes, washed $8+$ times with $1 \mathrm{X}$ PBS, and finally treated with 1:500 alexafluor 488-conjugated goat anti rabbit secondary antibody and tetrarhodamine isothiocyanate-conjugated wheat germ agglutinin (to stain cell walls) for 30 minutes at room temperature in the dark. Cells were visualized using an Olympus BX51 microscope equipped with a 100X brightfield objective and an Orca ER camera (Hammatsu). Length-per-ring ratios were calculated by measuring the total length of cells and counting FtsZ rings using Openlabs image analysis software.

\section{Quantitative immunoblotting}

Quantitative immunoblotting was performed essentially as described in Weart and Levin, 2003 [4], with the following modifications. To concentrate cells, $20 \mathrm{ml}$ of culture at an $\mathrm{OD}_{600}$ of 0.2 was pelleted and resuspended in $500 \mu \mathrm{l} \mathrm{PBS}$ in tubes containing $\sim 70 \mathrm{mg}$ silica beads. Cell lysates were prepared by bead beating in a FastPrep (MD Biomedical) machine for 2 pulses of 20 seconds each at $6.0 \mathrm{~m} / \mathrm{s}$. Gel loading was normalized to the sampling $\mathrm{OD}_{600}$. Ponceau $\mathrm{S}$ staining after the transfer was used as a loading control to normalize FtsZ staining to total protein. FtsZ was visualized using a 1:5,000 dilution of affinity purified rabbit antisera raised against $E$. coli FtsZ, the kind gift of David Weiss, and a secondary antibody conjugated to Horse Radish Peroxidase (Jackson ImmunoResearch Laboratories, West Grove, Pa). Quantification of FtsZ-bands and Ponceau staining was performed with ImageJ software. All quantifications were performed in the linear range of detection for the samples and antibodies. Note that using this approach we are able to reliably detect differences in intracellular FtsZ concentration between $10 \%$ and $15 \%$ [26].

\section{Protein purification}

FtsZ variants were cloned in the pET-21b $(+)$ expression vector using the $E$. coli strain AG1111. The resulting purified plasmids were freshly transformed into C41(DE3) cells [10] and consequently used for the expression of proteins. Briefly, 1 liter of LB medium was inoculated 1:100 with an overnight culture started from a single colony. Cells were grown at $37^{\circ} \mathrm{C}$ until $\mathrm{OD}_{600}$ was $\sim 0.6$ after which $f t s Z$ expression was induced with $1 \mathrm{mM}$ IPTG (isopropyl 1-thio- $\beta$-D-galactopyranoside). Cells were grown for an additional 4 hours at $37^{\circ} \mathrm{C}$ and then cells were harvested by centrifugation and pellets were stored at $-80{ }^{\circ} \mathrm{C}$. To purify FtsZ, frozen cell pellets were thawed on ice and resuspended in $30 \mathrm{~mL}$ induction buffer $(50 \mathrm{mM}$ Tris, pH 8.8, $100 \mathrm{mM} \mathrm{NaCl}, 1 \mathrm{mM}$ EDTA) with $1 \mathrm{mM}$ AEBSF (4-(2-Aminoethyl) benzenesulfonyl fluoride hydrochloride). Proteins were subjected to two rounds of lysis on a French press at 10,000 psi and the lysate clarified by spinning at 160,000xg for 45 minutes at $4{ }^{\circ} \mathrm{C}$.

FtsZ was precipitated from the supernatant with ammonium sulfate. In all cases, 0.25 of the volume of saturated ammonium sulfate was added to bring solution to a final concentration $20 \%$. Samples were incubated on ice for 20 minutes and spun at $10,000 x g$ for 10 minutes at $4{ }^{\circ} \mathrm{C}$. FtsZ84, FtsZ84*F39L, FtsZ84*M206I, and FtsZ84*V293I all precipitated in the first ammonium sulfate cut (20\% final concentration ammonium sulfate). For wild-type FtsZ, the supernatant was transferred to a new tube and 0.14 times the volume of saturated ammonium sulfate was added to bring the final concentration of ammonium sulfate to $30 \%$. The sample was incubated on ice for 20 minutes and spun at 10,000xg for 10 minutes at $4{ }^{\circ} \mathrm{C}$.

In all cases, pellets containing the bulk of the FtsZ protein were resuspended in low salt anion exchange buffer (50 mM Tris, pH 8.5, $50 \mathrm{mM} \mathrm{KCl,} 1$ mM EGTA, $10 \%$ sucrose) and further purified on a MT-20 column manually packed with UNOsphere ${ }^{\mathrm{mm}} \mathrm{Q}$ media with a linear gradient of $50-500 \mathrm{mM} \mathrm{KCl}$ in $50 \mathrm{mM}$ Tris, $\mathrm{pH} 8.5$, $1 \mathrm{mM}$ EGTA, $10 \%$ sucrose. Peak fractions were analyzed by SDS-page, pooled, and dialyzed overnight in 1 liter of FtsZ dialysis buffer, $\mathrm{pH} 7.5$ (50 mM HEPES, $\mathrm{pH} 7.5,50 \mathrm{mM} \mathrm{KCl}, 2.5 \mathrm{mM} \mathrm{MgCl} 2,1 \mathrm{mM}$ EGTA, $10 \%$ sucrose). FtsZ84 and FtsZ84* proteins were released from the column in a wider concentration of $\mathrm{KCl}$ than FtsZ and the FtsZ* proteins. Protein preparations were concentrated using PEG, aliquoted, flash frozen with liquid nitrogen, and stored at $-80{ }^{\circ} \mathrm{C}$.

\section{$90^{\circ}$ angle light scattering assay}

Light scattering assays were performed as previously described $[10,29]$ using a DM-45 spectrofluorimeter (Olis). Readings were taken every 0.25 seconds and a baseline reading was established for $60-100$ seconds before addition of $1 \mathrm{mM}$ GTP. Assembly reactions contained $5 \mu \mathrm{M}$ FtsZ in standard assembly buffer (50 mM MES, pH 6.5, $50 \mathrm{mM}$ $\mathrm{KCl}, 2.5 \mathrm{mM} \mathrm{MgCl}, 1 \mathrm{mM}$ EGTA) or in a high $\mathrm{Mg}^{++}$ buffer that instead contained $10 \mathrm{mM} \mathrm{MgCl}_{2}$. Data were 
collected by SpectralWorks (Olis) and exported into Microsoft Excel for processing.

\section{Electron microscopy}

Electron microscopy was performed as described [1, 2, 49]. FtsZ was assembled with GTP as for light scattering in the standard buffer (50 mM MES, pH 6.5, $50 \mathrm{mM} \mathrm{KCl,}$ $2.5 \mathrm{mM} \mathrm{MgCl} 2,1 \mathrm{mM}$ EGTA) or a buffer that instead had $250 \mathrm{mM} \mathrm{KCl} 5 \mu \mathrm{M}$ FtsZ was used for all in vitro assembly experiments. Samples were visualized using a JEOL 1200EX transmission electron microscope.

\section{GTPase assay}

GTPase activity was measured as previously described $[2,10]$, using the continuous, regenerative coupled GTPase assay of Ingerman and Nunnari [2, 8, 50]. Briefly, assays were conducted in buffer conditions identical to the standard buffer used for light scattering with $5 \mu \mathrm{M}$ FtsZ, $1 \mathrm{mM}$ GTP, $1 \mathrm{mM}$ phosphoenolpyruvate, $250 \mu \mathrm{M}$ NADH, 80 units/ml lactose dehydrogenase and 80 units $/ \mathrm{ml}$ pyruvate kinase. GTPase assays were performed at $30^{\circ} \mathrm{C}$. We used the linear decline of absorbance for NADH at $340 \mathrm{~nm}$ for $3 \mathrm{~min}$ in a quartz cuvette (1-cm path length) using a SPECTRAmax Plus spectrophotometer (Molecular Devices). The raw data of absorbance per minute was converted to activity using the extinction coefficient for $\mathrm{NADH}$ of $6220 \mathrm{M}^{-1} \mathrm{~cm}^{-1}$. GTPase data are the average of $3-5$ independent experiments.

\section{Kinetics curves}

Kinetics curves were generated by performing GTPase assays as in the previous paragraph while varying the GTP concentrations from 0.1 to $6 \mathrm{mM}$ (all kinetics data obtained at $22{ }^{\circ} \mathrm{C}$ using an Eon spectrophotometer (BioTek). At least 5 GTP concentrations were used per FtsZ mutant and values used to calculate the $\mathrm{K}_{\mathrm{m}}$ and $\mathrm{V}_{\max }$ were the average of at least 3 replicates per GTP concentration. The $\mathrm{K}_{\mathrm{m}}$ and $\mathrm{V}_{\max }$ were calculated using Sigma Plot software. The best fit for the data was obtained using the Hill-modified Michaelis-Menten equation: $\mathrm{V}_{0}=$ $\mathrm{V}_{\max }[\mathrm{S}]^{\mathrm{n}} /\left(\mathrm{K}_{\mathrm{m}}^{\mathrm{n}}+[\mathrm{S}]^{\mathrm{n}}\right)$.

\section{zapA deletion and overexpression experiments}

$z a p A$ deletions and overexpression strains were generated utilizing standard genetic procedures (transduction and transformation) discussed above [18]. The zapA deletion strain from the Keio collection was acquired through the E. coli stock center at Yale and was used as a donor for phage transduction into our recipient wild-type, ftsZ84, and ftsZ84* strains. The sodium salicylate inducible zapA plasmid (pKG110-zapA) (gift of Bill Margolin) and the IPTG inducible zapA plasmid (pQE80-H6-zapA) (gift of Alex Dajkovic) were transformed into the wild-type and ftsZ84 background strains [17, 51]. For the plating efficiency assays (see above), all strains were grown in LB-1 \% NaCl to an $\mathrm{OD}_{600}$ of 0.2 and plated onto LB-no salt plates (zapA deletion strains) or LB-no salt ampicillin plates with $0,0.1 \mu \mathrm{M}$, or $1.0 \mu \mathrm{M}$ sodium salicylate.

\section{Availability of supporting data}

All supporting data are included in the following additional file to the manuscript:

Additional file 1, Adobe Acrobat .pdf format, zapA overexpression does not restore colony formation to ftsZ84 cells under nonpermissive conditions, this file shows colony forming data of ftsZ84 cells when the zapA gene is induced.

\section{Ethics statement}

Ethics approval was not required for this study, which utilized only bacteria and did not involve humans, human data or animals.

\section{Additional file}

Additional file 1: zapA overexpression does not restore colony

formation to ftsZ84 cells under nonpermissive conditions. (PDF 807 kb)

\section{Abbreviations}

LB: Lysogeny broth; OD: Optical density; L/R: Length per ring; EMS: Ethylmethanesulfonate; EM: Electron microscopy.

Competing interests

The authors declare that they have no competing interests.

\section{Authors' contributions}

HA designed and performed all aspects of the experimentation except as noted below, analyzed data, and drafted the manuscript. BL and EE performed the mutant screens to identify intragenic suppressors of ftsZ84 and performed the in vitro analysis in Fig. 5 and the GTPase assays. AS made the zapA deletion and overexpression strains and performed the plating efficiency in Fig. 6. PAL helped design experiments and draft the manuscript. All authors read and approved the final manuscript.

\section{Acknowledgements}

We would like to thank all members of the Levin Lab and the Zaher lab for useful discussions during the course of this research. We are particularly grateful to Corey Westfall for helping with the structural analysis of the FtsZ84* mutations and expertise in enzyme kinetics and Marcus Kwon for help with plating efficiency assays. We would also like to thank Joe Jez, Anuradha Janakiraman and PJ Buske for insightful comments on the manuscript. We are indebted to David S. Weiss for the E. coli FtsZ antiserum used in this work, to Jon Beckwith, Alex Dajkovic, Bill Margolin, Debabrata RayChaudhuri, for strains and other reagents, and to George Weinstock and the Genome Institute at Washington University in St. Louis for assistance with genome sequencing and analysis. This work was supported by National Institutes of Health grants GM64671 to PAL, T32 GM007067 to HAA and a Washington University STARS fellowship to ERE.

\section{Author details}

'Department of Biology, Washington University in St. Louis, St. Louis, MO 63130, USA. ${ }^{2}$ Present address: Department of Bioengineering, Stanford University, Stanford, CA 94305, USA.

Received: 12 May 2015 Accepted: 2 October 2015

Published online: 13 October 2015 


\section{References}

1. Adams DW, Errington J. Bacterial cell division: assembly, maintenance and disassembly of the Z ring. Nat Rev Microbiol. 2009;7:642-53.

2. Erickson HP, Anderson DE, Osawa M. FtsZ in bacterial cytokinesis: cytoskeleton and force generator all in one. Microbe. 2010;74:504-28.

3. Den Blaauwen T, Buddelmeijer N, Aarsman ME, Hameete CM, Nanninga N. Timing of FtsZ assembly in Escherichia coli. J Bacteriol. 1999;181:5167-75.

4. Weart RB, Levin PA. Growth rate-dependent regulation of medial FtsZ ring formation. J Bacteriol. 2003;185:2826-34.

5. Errington J, Daniel RA, Scheffers D-J. Cytokinesis in bacteria. Microbe. 2003;67:52-65.

6. Aarsman MEG, Piette A, Fraipont C, Vinkenvleugel TMF, Nguyen-Distèche $M$, Blaauwen Den T. Maturation of the Escherichia coli divisome occurs in two steps. Mol Microbiol. 2005;55:1631-45.

7. Levin P, Schwartz R, Grossman A. Polymer stability plays an important role in the positional regulation of FtsZ. J Bacteriol. 2001;183:5449-5452.

8. Scheffers D-J, De Wit JG, Blaauwen Den T, Driessen AJM. GTP hydrolysis of cell division protein FtsZ: evidence that the active site is formed by the association of monomers. Biochemistry. 2002;41:521-9.

9. Mukherjee A, Lutkenhaus J. Guanine nucleotide-dependent assembly of FtsZ into filaments. J Bacteriol. 1994;176:2754-8.

10. Buske PJ, Levin PA. Extreme $C$ terminus of bacterial cytoskeletal protein FtsZ plays fundamental role in assembly independent of modulatory proteins. J Biol Chem. 2012;287:10945-57.

11. Bi E, Lutkenhaus J. Analysis of $\mathrm{fts} Z$ mutations that confer resistance to the cell division inhibitor SulA (SfiA). J Bacteriol. 1990;172:5602-9.

12. Hirota $Y$, Ryter A, Jacob F. Thermosensitive mutants of $E$. coli affected in the processes of DNA synthesis and cellular division. Cold Spring Harb Symp Quant Biol. 1968:33:677-93.

13. Addinall SG, Bi E, Lutkenhaus J. FtsZ ring formation in fts mutants. J Bacteriol. 1996;178:3877-84.

14. RayChaudhuri D, Park JT. Escherichia coli cell-division gene fts $Z$ encodes a novel GTP-binding protein. Nature. 1992;359:251-4.

15. De Boer P, Crossley R, Rothfield L. The essential bacterial cell-division protein FtsZ is a GTPase. Nature. 1992;359:254-6.

16. Lu C, Stricker J, Erickson H. Site-specific mutations of FtsZ- effects on GTPase and in vitro assembly. BMC Microbiol. 2001;1:7.

17. Dajkovic A, Pichoff S, Lutkenhaus J, Wirtz D. Cross-linking FtsZ polymers into coherent $Z$ rings. Mol Microbiol. 2010;78:651-68.

18. Miller JH. A Short Course in Bacterial Genetics - A Laboratory manual and handbook for Escherichia coli and related bacteria. Cold Spring Harbor. 1992. http://www.amazon.com/A-Short-Course-Bacterial-Genetics/dp/0879693495.

19. Kohiyama M, Cousin D, Ryter A, Jacob F. Mutants Thermosensibles D'Escherichia coli K 12. Annales de L'institute Pasteur. 1966;110:466-86.

20. Boyle DS, Khattar MM, Addinall SG, Lutkenhaus J, Donachie WD. ftsW is an essential cell-division gene in Escherichia coli. Mol Microbiol. 2003;24:1263-73.

21. Mercer KLN, Weiss DS. The Escherichia coli cell division protein FtsW is required to recruit its cognate transpeptidase, Ftsl (PBP3), to the division site. J Bacteriol. 2002;184:904-12.

22. Michaud C, Mengin-Lecreulx D, van Heijenoort J, Blanot D. Over-production, purification and properties of the uridine-diphosphate-N-acetylmuramoyl-Lalanyl-D-glutamate: meso-2,6-diaminopimelate ligase from Escherichia coli. Eur J Biochem. 1990;194:853-61.

23. Arjes HA, Kriel A, Sorto NA, Shaw JT, Wang JD, Levin PA. Failsafe Mechanisms Couple Division and DNA Replication in Bacteria. Curr Biol. 2014;24:2149-55.

24. Powell BS, Court DL. Control of fts Z expression, cell division, and glutamine metabolism in Luria-Bertani medium by the alarmone ppGpp in Escherichia coli. J Bacteriol. 1998;180:1053-62.

25. Camberg JL, Hoskins JR, Wickner S. ClpXP protease degrades the cytoskeleta protein, FtsZ, and modulates FtsZ polymer dynamics. Proc Natl Acad Sci U S A. 2009;106:10614-9.

26. Hill NS, Buske PJ, Shi Y, Levin PA. A moonlighting enzyme links Escherichia coli cell size with central metabolism. PLoS Genet. 2013;9:e1003663.

27. Potluri L-P, de Pedro MA, Young KD. Escherichia coli low-molecular-weight penicillin-binding proteins help orient septal FtsZ, and their absence leads to asymmetric cell division and branching. Mol Microbiol. 2012;84:203-24.

28. Anderson D, Kim M, Moore J, O'Brien T, Sorto N, Grove C, et al. Comparison of Small Molecule Inhibitors of the Bacterial Cell Division Protein FtsZ and Identification of a Reliable Cross-Species Inhibitor. ACS Chem Biol. 2012:7:1918-28.
29. Haeusser DP, Garza AC, Buscher AZ, Levin PA. The division inhibitor EzrA contains a seven-residue patch required for maintaining the dynamic nature of the medial FtsZ ring. J Bacteriol. 2007;189:9001-10.

30. Sossong TM, Brigham-Burke MR, Hensley P, Pearce KH. Self-activation of guanosine triphosphatase activity by oligomerization of the bacterial cell division protein FtsZ. Biochemistry. 1999:38:14843-50.

31. Salvarelli E, Krupka M, Rivas G, Vicente M, Mingorance J. Independence between GTPase active sites in the Escherichia coli cell division protein FtsZ. FEBS Lett. 2011;585:3880-3.

32. Miraldi ER, Thomas PJ, Romberg L. Allosteric models for cooperative polymerization of linear polymers. Biophys J. 2008;95:2470-86.

33. Mukherjee A, Lutkenhaus J. Analysis of FtsZ assembly by light scattering and determination of the role of divalent metal cations. J Bacteriol. 1999:181:823-32.

34. Mukherjee A, Saez C, Lutkenhaus J. Assembly of an FtsZ mutant deficient in GTPase activity has implications for FtsZ assembly and the role of the $Z$ ring in cell division. J Bacteriol. 2001;183:7190-7.

35. Gueiros-Filho FJ, Losick R. A widely conserved bacterial cell division protein that promotes assembly of the tubulin-like protein FtsZ. Genes Dev. 2002:16:2544-56.

36. Buske PJ, Levin PA. A flexible C-terminal linker is required for proper FtsZ assembly in vitro and cytokinetic ring formation in vivo. Mol Microbiol. 2013;89(2):249-63.

37. Yu XC, Margolin W. Ca2 + -mediated GTP-dependent dynamic assembly of bacterial cell division protein FtsZ into asters and polymer networks in vitro. EMBO J. 1997;16:5455-63.

38. Mendieta J, Rico Al, López-Viñas E, Vicente M, Mingorance J, Gómez-Puertas P. Structural and functional model for ionic $(\mathrm{K}(+) / \mathrm{Na}(+))$ and $\mathrm{pH}$ dependence of GTPase activity and polymerization of FtsZ, the prokaryotic ortholog of tubulin. J Mol Biol. 2009;390:17-25.

39. Buss J, Coltharp C, Huang T, Pohlmeyer C, Wang S-C, Hatem C, et al. In vivo organization of the FtsZ-ring by ZapA and ZapB revealed by quantitative super-resolution microscopy. Mol Microbiol. 2013;89:1099-120.

40. Mingorance J, Rueda S, Gómez-Puertas P, Valencia A, Vicente M. Escherichia coli FtsZ polymers contain mostly GTP and have a high nucleotide turnover. Mol Microbiol. 2001:41:83-91.

41. Huecas S, Llorca O, Boskovic J, Martín-Benito J, Valpuesta JM, Andreu JM. Energetics and geometry of FtsZ polymers: nucleated self-assembly of single protofilaments. Biophys J. 2008;94:1796-806.

42. Rico Al, Krupka M, Vicente M. In the beginning, Escherichia coli assembled the proto-ring: an initial phase of division. J Biol Chem. 2013;288:20830-6.

43. Chen $Y$, Erickson HP. Rapid in vitro assembly dynamics and subunit turnover of FtsZ demonstrated by fluorescence resonance energy transfer. J Biol Chem. 2005;280:22549-54.

44. Haeusser DP, Rowlett W, Margolin W. A mutation in Escherichia coli ftsZ bypasses the requirement for the essential division gene zipA and confers resistance to FtsZ assembly inhibitors by stabilizing protofilament bundling. Mol Microbiol. 2015;97(5):988-1005.

45. Addinall S, Cao C, Lutkenhaus J. Temperature shift experiments with an ftsZ84(Ts) strain reveal rapid dynamics of FtsZ Localization and Indicate that the Z Ring Is Required throughout Septation and Cannot Reoccupy Division Sites Once Constriction Has Initiated. J Bacteriol. 1997;1997:4277-84.

46. Weiss DS, Chen JC, Ghigo J-M, Boyd D, Beckwith J. Localization of FtsI (PBP3) to the Septal Ring Requires Its Membrane Anchor, the Z Ring, FtsA, FtsQ, and FtsL. J Bacteriol. 1999;181:508-20

47. Sambrook J, David W. Molecular Cloning: a Laboratory Manual. 3rd ed. Cold Spring Harbor: Cold Spring Harbor Laboratory; 2001.

48. Levin PA. Light Microscopy Techniques for Bacterial Cell Biology. Methods in Microbiology. 2002;31:115-32.

49. Weart RB, Lee AH, Chien A-C, Haeusser DP, Hill NS, Levin PA. A metabolic sensor governing cell size in bacteria. Cell. 2007;130:335-47.

50. Ingerman E, Nunnari J. A continuous, regenerative coupled GTPase assay for dynamin-related proteins. Meth Enzymol. 2005;404:611-9.

51. Haeusser DP, Hoashi M, Weaver A, Brown N, Pan J, Sawitzke JA, et al. The Kil peptide of bacteriophage $\lambda$ blocks Escherichia coli cytokinesis via ZipA-dependent inhibition of FtsZ assembly. PLoS Genet. 2014;10:e1004217.

52. Matsui T, Han X, Yu J, Yao M, Tanaka I. Structural change in FtsZ Induced by intermolecular interactions between bound GTP and the T7 loop. J Biol Chem. 2014:289:3501-9. 